\title{
Decreased expression of peroxiredoxin I inhibits proliferation, invasion, and metastasis of ovarian cancer cell
}

This article was published in the following Dove Press journal:

OncoTargets and Therapy

\author{
Ming-Jun Zheng ${ }^{1,2, *}$ \\ Jing Wang ${ }^{1,2, *}$ \\ Hui-Min Wang ${ }^{1,2}$ \\ Ling-Ling Gao',2 \\ Xiao $\mathrm{Li}^{1,2}$ \\ Wen-Chao Zhang ${ }^{1,2}$ \\ Rui Gou ${ }^{1,2}$ \\ Qian Guo ${ }^{1,2}$ \\ Xin $\mathrm{Nie}^{1,2}$ \\ Juan-Juan Liu',2 \\ Bei $\operatorname{Lin}^{1,2}$
}

'Department of Gynaecology and Obstetrics, Shengjing Hospital

Affiliated to China Medical University,

Heping District, Shenyang I 10004 ,

Liaoning, China; ${ }^{2}$ Key Laboratory

of Maternal-Fetal Medicine of

Liaoning Province, Key Laboratory of

Obstetrics and Gynecology of Higher

Education of Liaoning Province,

Liaoning, China

*These authors contributed equally to this work

\begin{abstract}
Aim: The aim of this study was to explore the expression of peroxiredoxin1 (PRDX1) in epithelial ovarian cancer, analyze the relationship between PRDX1 and clinicopathologic parameters of patients with ovarian cancer, including their prognosis, and describe changes and the mechanisms involved in malignant biologic behavior of ovarian cancer cells when PRDX1 expression is inhibited.
\end{abstract}

Methods: The expression of PRDX1 was detected immunohistochemically in 15 samples of normal ovarian tissue, 21 benign, 11 borderline, and 101 malignant epithelial ovarian tumors. Changes in ovarian cancer cell proliferation, invasion, and metastasis before and after inhibiting PRDX1 expression were assessed by cell function assay. Additionally, gene set enrichment analysis (GSEA) of PRDX1 was performed by the Cancer Genome Atlas database. A proteinprotein interaction network was then constructed and a pathway function analysis of the genes in the network was conducted.

Results: PRDX1 expression was mainly localized to the cytoplasm, as well as the nucleus of cells. The expression rate of PRDX1 in epithelial ovarian malignant tissues (96.04\%) was significantly higher than that in borderline $(72.72 \%)$ and benign $(57.14 \%)$ epithelial ovarian tumors, and normal ovarian tissue $(20 \%$; all $P<0.05)$. Cox multivariate regression analysis indicated that advanced clinical stage, low tissue differentiation, and high expression of PRDX1 were independent risk factors affecting the prognosis of epithelial ovarian cancer (all $P<0.05$ ). Cell function assay verified that the decreased expression of PRDX1 inhibited ovarian cancer cell proliferation, invasion, and metastasis. GSEA analysis indicated that PRDX1 was significantly related to the Wnt signaling pathway. Western blot analysis confirmed that PRDX1 could regulate the expression of $\beta$-catenin in the Wnt pathway.

Conclusion: Decreased expression of PRDX1 can attenuate cell proliferation, invasion, and metastasis of ovarian cancer cells. The expression of PRDX1 is related to the prognosis of patients with ovarian cancer and can therefore be used as a biomarker.

Keywords: epithelial ovarian cancer, peroxiredoxin1, immunohistochemistry, invasion, metastasis, pathways

\section{Introduction}

Ovarian cancer is one of three malignant tumors of the female genital tract. Due to the absence of early warning symptoms and the lack of effective diagnostics, about $70 \%$ of cases are diagnosed only at an advanced stage. Invasion and metastasis are the most common causes of mortality for patients with ovarian cancer and the prognosis is relatively poor. ${ }^{1,2}$ So, it is of great importance to explore the mechanism(s) behind the 
development of ovarian cancer and to identify effective tumor biomarkers in an effort to improve the prognosis of this insidious disease.

Peroxiredoxins (PRDXs), first described in yeast, were found to be widespread in other organisms after follow-up studies. ${ }^{3}$ PRDX is a family of redox regulating proteins that eliminate ROS and maintain the stability of hydrogen peroxide $\left(\mathrm{H}_{2} \mathrm{O}_{2}\right)$ in cells. The mammalian PRDX family is made up of six members, from PRDX1 to PRDX6, which can be divided into three subgroups based on the number of cysteine residues and the catalytic reaction mechanism: typical 2-cysteine PRDX1-4, atypical 2-cysteine PRDX5, and 1-cysteine PRDX6 proteins. ${ }^{4}$ PRDX1 contains conserved $\mathrm{N}$-terminal $\mathrm{Cys}^{52}$ and C-terminal Cys ${ }^{173}$ sequences. ${ }^{5} \mathrm{An}$ antioxidant, PRDX1 regulates cell growth, differentiation, and apoptosis. Within the nucleus, PRDX1 affects bioactivities upon gene regulation by associating with $\mathrm{p} 53$ or various transcription factors, and then induces or suppresses cell death. Additionally, PRDX1 in the cytoplasm shows anti-apoptotic potential through interactions with an ROSdependent signaling pathway. ${ }^{6}$ In recent studies, PRDX1 was found to be abnormally expressed in esophageal, ${ }^{7}$ lung, ${ }^{8}$ and breast tumor cells ${ }^{9}$ and was also found to be overexpressed in gynecological malignancies such as ovarian, ${ }^{10}$ endometrial, ${ }^{11}$ and cervical cancers. ${ }^{12}$ However, recent research has mainly focused on the roles of PRDX1 in the evaluation of clinical prognoses. In addition, literature regarding its functional mechanisms, especially its roles in malignant biologic behaviors of ovarian cancer cells, is scarce.

Detecting the expression of PRDX1 in ovarian tumor cells by immunohistochemistry (IHC) and evaluating the biologic behaviors of ovarian malignant tumor cells, before and after PRDX1 transfection, by cell function assays will provide a theoretical basis for the early diagnosis and targeted therapy of ovarian cancer. At the same time, the gene set enrichment analysis (GSEA) of PRDX1 with the Cancer Genome Atlas (TCGA) public database, pathway functional analysis of genes associated with PRDX1 will provide clues for exploring the roles and mechanisms of PRDX1 in the development of ovarian cancer.

\section{Materials and methods}

\section{Participants and specimens}

Ovarian samples were collected from 148 surgical patients hospitalized at the Department of Gynecology of our hospital from 2008 to 2012. All the patients were informed about the experiments and signed informed consent was obtained. The tissues-associated experiments were approved by the
Clinical Research Ethics Committee of Shengjing Hospital affiliated to China Medical University. The median ages of patients who supplied samples of malignant, borderline, and benign tumors, as well as normal ovaries were 54 (19-77), 62 (49-74), 58.7 (36-74), and 43 (35-65) years, respectively. With regard to age, a significant statistical difference between groups was not noted $(P>0.05)$. Samples were embedded in paraffin and all pathological sample sections were examined by experienced pathologists. The 148 samples included those from 101 malignant tumors originating from ovarian epithelia (including 55 cases of serous carcinoma, 14 cases of endometrioid carcinoma, four cases of mucinous carcinoma, eight cases of clear cell carcinoma, and 20 poorly differentiated adenocarcinoma), 11 epithelial ovarian borderline tumors, 21 epithelial ovarian benign tumors, and 15 normal ovarian tissues. Of the ovarian cancer samples, 20 were well differentiated, 24 were moderately differentiated, and 57 were poorly differentiated in terms of histological classification. Based on the 2009 International Federation of Gynecology and Obstetrics (FIGO) staging system, there were 39 cancer cases at stages I-II and 62 cases at stages III-IV. Twenty-nine cases had pelvic and/or para-aortic lymph node metastases, 65 had no metastasis, and seven cases did not undergo lymph node screening before surgery. Patients were not treated with radiotherapy, chemotherapy, or hormone therapy.

All procedures performed in the studies involving human participants were in accordance with the ethical standards of the institutional and/or national research committee and with the 1964 Helsinki Declaration and its later amendments or comparable ethical standards.

\section{Immunohistochemistry}

Each group of ovarian samples was fixed in $10 \%$ formalin, embedded in paraffin, and processed as $5 \mu \mathrm{m}$ continuous sections. Samples were dewaxed with discontinuous concentrations of ethanol, and blocked to inhibit endogenous peroxidase. They were then heated in a microwave to retrieve antigens, cooled to room temperature, and blocked by incubation in goat serum for 30 minutes at $37^{\circ} \mathrm{C}$. Samples were incubated in rabbit anti-PRDX1 (Abcam, Cambridge, UK; $1: 1,200$ ) overnight at $4^{\circ} \mathrm{C}$, followed by incubation with horseradish peroxidase-coupled goat anti-rabbit secondary antibody at $37^{\circ} \mathrm{C}$ for 30 minutes, and stained by 3,3'-diaminobenzidine. The cell nucleus was stained blue by hematoxylin. Sections were then dehydrated, cleared by xylene, and mounted. PRDX1 expression was detected by IHC using a streptavidin peroxidase method. PRDX1 expression in liver was used as a positive control. Samples incubated 
with PBS instead of PRDX1 primary antibody were used as a negative control. Positive and negative controls were included for each batch of immunohistochemically stained sections. The experimental procedure was performed by strictly following the manufacturer's instructions.

\section{Quantification of immunohistochemical staining}

An immunohistochemical result was considered positive if brownish yellow staining was found in the cytosol. Staining results were classified as negative, light yellow, brownish yellow, and dark brown according to the staining density and scored $0,1,2$, or 3, respectively. This is called score 1 . Five fields under the microscope at $400 \times$ magnification were randomly selected and scored for each section. The average score represented the percentage of positive cells in a field of view under the microscope. All sections were examined and scored $0,1,2,3$, or 4 if the positive cell percentage was $<5 \%, \geq 5 \%$ and $\leq 25 \%, \geq 26 \%$ and $\leq 50 \%$, $\geq 51 \%$ and $\leq 75 \%$, and $>75 \%$, respectively. This was called score 2 . Score $3=$ score $1 \times$ score 2 . The staining result was considered negative $(-)$, weak positive $(+)$, moderate positive $(++)$, and strong positive $(+++)$ if score 3 fell between 0 and 2, 3 and 4 , 5 and 8 , and 9 and 12, respectively. To control the reading variance, each section was read by two different observers. A third observer arbitrated if the two observers' reading results were not consistent.

\section{Kaplan-Meier (KM) plotter}

KM plotter (http://kmplot.com) has assessed the effect of 54,675 genes on survival using 10,188 cancer samples, including 4,142 breast, 1,648 ovarian, 2,437 lung, and 1,065 gastric cancer patients. Ovarian cancer patients were divided into two groups according to the expression of a certain gene (high expression and low expression). KM plots were used to analyze overall survival in patients with ovarian cancer. The HR with $95 \% \mathrm{CIs}$ and $\log$-rank $P$-value were displayed online.

\section{Oncomine database extraction}

The Oncomine database is currently the world's largest oncogene chip database and integrated data mining platform for the purpose of mining cancer gene information. To date, the database has collected 715 gene expression data sets, and 86,733 cancer tissue and normal tissue sample data. The Oncomine database has been applied for differential expression classification for common cancer types, and their respective normal tissues, as well as clinical and pathological analysis.

Enter "PRDX1" in the Oncomine database, "Ovarian Cancer" for cancer type, and set the threshold $(P$-value $<0.05$, gene rank=top 10\%). After comparing, the expression of PRDX1 in multiple ovarian cancer data sets is obtained; further, "Multi-Cancer Panel Data sets" is selected to obtain differential expression of this gene in various types of cancer tissues.

\section{Analysis of copy number variation}

The ovarian cancer copy number variation data were downloaded from cBioPortal (http://www.cbioportal.org/), and the samples were divided into three groups according to copy number deletion (loss), diploid (diploid), and copy number increase (gain). $t$-test was used to compare the expression levels of PRDX1.

\section{Analysis of methylation level and the expression level of PRDXI}

The ovarian cancer methylation data were downloaded from Xena (https://xenabrowser.net/datapages/), and the correlation between the methylation level and the expression level of $P R D X 1$ gene was tested by Pearson's correlation analysis

\section{Construction of expression vectors, cell culture, and PRDXI transfection}

Human epithelial ovarian cancer cells lines Caov-3 were purchased from the American Type Culture Collection (ATCC, Manassas, VA, USA) and cultured in RPMI-1640 containing 10\% FBS. Cells were transfected using liposomes with a vector transfection kit according to the instructions. Three small interfering RNA (siRNA) expression vectors for human PRDX1 were constructed using the vector pSilence. The mRNA target sequences chosen for designing PRDX1siRNA are GCA CCA UUG CUC AGG AUU ATT for PRDX1-siRNA1, GCU CUG UGG AUG AGA CUU UTT for PRDX1-siRNA2, and CCA GUU CAC UGA CAA ACA UTT for PRDX1-siRNA3. At 48 hours after transfection, the transfection efficiency of PRDX1 was identified by Western blot analyses.

\section{Transfection identification by Western blot}

Total protein extracts from ovarian cancer cells were separated by $12 \%$ SDS-PAGE and transferred overnight onto a methanol-activated polyvinylidene difluoride membrane. After blocking, the membrane was washed with $0.3 \%$ Tween-20 in Tris-buffered saline four times, 15 minutes each time, and 
incubated with PRDX1 primary antibody (Abcam; rabbit polyclonal, 1:300) overnight at $4^{\circ} \mathrm{C}$. The membrane was washed again and incubated with horseradish peroxidasecoupled secondary antibody (Santa Cruz Biotechnology, Inc, Dallas, TX, USA) at room temperature for 1 hour. Western blot results were visualized with ImageJ $1.31 \mathrm{v}$ (Wayne Rasband, Bethesda, Maryland, USA) and normalized to the GAPDH protein level.

\section{Malignant biological behavior testing of cells after PRDXI transfection \\ Cell proliferation assay}

An MTT assay was used to assess cell proliferation. MTT (50 mg) was dissolved in $10 \mathrm{~mL}$ PBS (0.01 mol/L, $\mathrm{pH}=7.4)$ to achieve a final concentration at $5 \mathrm{mg} / \mathrm{mL}$, sterilized by filtering, aliquoted, and stored at $4^{\circ} \mathrm{C}$ in the dark. Each sample was tested in triplicate. Cells were routinely trypsinized and counted, washed twice with PBS, and resuspended in complete culture medium without antibiotics to reach a cell concentration of $1 \times 10^{3} / 100 \mu \mathrm{L}$. A cell suspension $(200 \mu \mathrm{L})$ was added to each well on a 96-well plate (the plate was exposed to ultraviolet light for 4 hours prior to use) and cultured at $37^{\circ} \mathrm{C}, 5 \% \mathrm{CO}_{2}$ in the incubator for 24,48 , and 72 hours. At the indicated time points, the cell culture medium was removed, and $0.02 \mathrm{~mL}$ of a $5 \mathrm{mg} / \mathrm{mL}$ MTT solution was added to each well and incubated for 4 hours at $37^{\circ} \mathrm{C}$. After the 4-hour incubation, the supernatant was removed and $200 \mu \mathrm{L}$ dimethyl sulfoxide was added to each well. The plate was shaken on a flat shaker for 10 minutes, and then was read at $\mathrm{OD}_{490}$. The $\mathrm{OD}_{490}$ value for each well=raw $\mathrm{OD}_{490}$ for each well-background $\mathrm{OD}_{490}$ value (MTT solution without cells). Assays were repeated at least three times independently.

\section{Wound healing assay}

Exponentially growing cells were resuspended to a single cell suspension, seeded in wells on a 6 -well plate at $1 \times 10^{3}$ cells/mL, and allowed to grow to $90 \%$ confluency. A wound was made in the center of the monolayer of cells using a $200-\mu \mathrm{L}$ sterilized pipette tip. Cells were washed and cultured in medium without serum for 24 hours. Images of the cells that had migrated into the cell-free wound area were obtained and the migration distance was observed under a microscope. The scratch wound widths were calculated by the relative percentage compared to the untreated control cells.

\section{Transwell assay}

The membrane of the upper chamber of a transwell plate was covered with 1:8 diluted $50 \mathrm{mg} / \mathrm{L}$ Matrigel solution, air dried at $4^{\circ} \mathrm{C}$, and the residual solution removed. For each chamber, $50 \mu \mathrm{L}$ culture medium containing $10 \mathrm{~g} / \mathrm{L}$ BSA was added without any serum, and the chambers were kept in an incubator at $37^{\circ} \mathrm{C}, 5 \% \mathrm{CO}_{2}$ for 5 hours until the Matrigel solidified. Cells were trypsinized, centrifuged, washed twice, and resuspended to a single cell suspension with BSA containing culture medium (no serum).

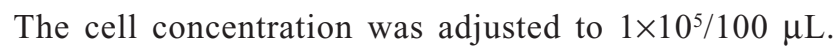
For the upper chamber, $100 \mu \mathrm{L}$ of cell suspension and $200 \mu \mathrm{L}$ BSA containing the culture medium (no serum) were added. For the lower chamber, $500 \mu \mathrm{L}$ culture medium containing 10\% FBS was added. The cells in the transwells were incubated for 24 hours and then the Matrigel and cells in the upper chamber were removed. The membrane in the upper chamber was inverted, fixed in methanol for 20 minutes, and stained by Trypan blue for 5 minutes. Five fields were randomly selected under the microscope and the cell number attached to the membrane was counted. An average number of five fields was calculated and used for statistical analysis. Each sample was tested in triplicate, and the experiment was repeated three times independently.

\section{Data collection from TCGA database}

Ovarian cancer data were downloaded, prescreened, and 374 tumor samples were included in this study. Samples were sorted according to the expression level of PRDX1, from low to high, and equally aliquoted into four parts. The first $25 \%$ of the samples constituted the PRDX1 low expression group. The last $25 \%$ of the samples formed the high expression group.

\section{Gene set enrichment analysis}

GSEA 3.0 was used to analyze data. ${ }^{13} \mathrm{C} 2$.cp.kegg.v6.1.symbols. gmt data cluster was downloaded from the Molecular Signatures Database data bank on the GSEA website. Enrichment analysis was performed on the sorted samples using default weighted enrichment statistics. Random assortment times were set to 1,000 .

\section{Functional analysis and signaling pathway enrichment analysis}

Proteins that interact with PRDX1 were obtained from GeneCloud of Biotechnology Information (GCBI; https://www. gcbi.com.cn/gclib/html/index) and the corresponding genes of those proteins were analyzed using DAVID database (https://david.ncifcrf.gov/). The DAVID database is an essential foundation for the success of any high-throughput gene 
function analysis. The functional and pathway enrichment of the proteins encoded by candidate genes were analyzed, and a $P$-value $<0.05$ was considered statistically significant.

\section{Construction of protein interaction network and module screening}

Protein-protein interaction network analysis was carried out with the following databases: BioGrid, InWeb_IM, and OmniPath. The resultant network contains the subset of proteins that undergo physical interactions with at least another list member. If the network contained 3-500 proteins, Molecular Complex Detection (MCODE) ${ }^{14}$ was further applied to identify densely connected network components. Biological process and pathway enrichment analysis were identified.

\section{Statistical analysis}

Data were analyzed by SPSS 19.0 software (IBM Corporation, Armonk, NY, USA). Chi-squared test and Fisher's exact test were used to analyze counting data, and $t$-test was used to analyze measurement data. KM and log-rank analyses were used to analyze survival curves. A Cox model was used to analyze relationships between data and patients. $P<0.05$ was considered to be statistically significant. For GSEA analysis, gene clusters with false discovery rates $<0.25$ and $P<0.05$ were considered as significantly enriched genes.
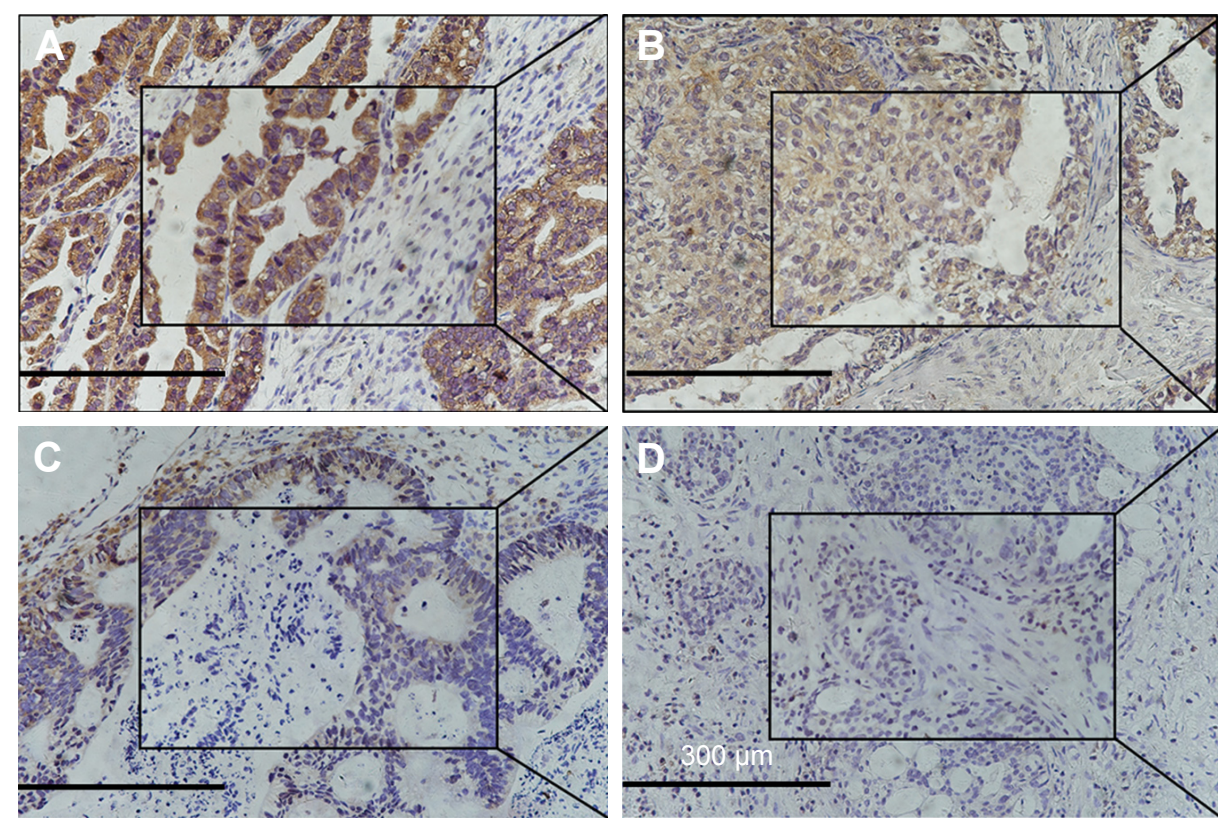

Figure I The expression of PRDXI in ovarian tissues (SP $\times 200$, central SP $\times 400$ ).

Notes: (A) Ovarian epithelial malignant tumor. (B) Ovarian epithelial borderline tumor. (C) Ovarian epithelial benign tumor. (D) Normal ovarian tissue. Scale bar: $300 \mu \mathrm{m}$. Abbreviations: PRDXI, peroxiredoxin I; SP, scaled pixels.

\section{Results \\ PRDXI is significantly upregulated in ovarian cancer and correlates with clinical characteristics}

To investigate the clinical values of PRDX1 in ovarian cancer, we performed IHC analysis of 148 ovarian tissue samples, including 15 samples of normal ovarian tissue, 21 benign, 11 borderline, and 101 malignant epithelial ovarian tumors.

The expression of PRDX1 was demonstrated by a brownish yellow color after immunohistochemical staining of tissue sections; it was mainly expressed not only in the cytosol but was also found in the nucleus. PRDX1 expression was significantly upregulated in ovarian cancer tissue compared with normal lung tissue (Figure 1).

The positive and high positive rates of PRDX1 in epithelial ovarian cancer were $96.04 \%$ and $84.16 \%$, respectively, which were significantly higher than those in epithelial ovarian borderline tumors (72.72\% and $27.27 \%$ ), benign epithelial ovarian tumors (57.14\% and $14.29 \%$ ), and normal ovarian tissues ( $20 \%$ and $0 \%$ ) (all $P<0.05$; Table 1$)$. Although these rates were higher in epithelial ovarian borderline tumors than in benign tumors and, in turn, were higher in benign tumors than in normal tissue, a significant difference between any two out of these three groups mentioned above was not found ( $P>0.05$ for each comparison; Table 1). 
Table I Expression of PRDXI in ovarian tissues

\begin{tabular}{|c|c|c|c|c|c|c|c|}
\hline \multirow[t]{2}{*}{ Group } & \multirow[t]{2}{*}{$\mathbf{n}$} & \multicolumn{2}{|c|}{ Low } & \multicolumn{2}{|c|}{ High } & \multirow{2}{*}{$\begin{array}{l}\text { Positive } \\
\text { rate (\%) }\end{array}$} & \multirow{2}{*}{$\begin{array}{l}\text { High } \\
\text { positive } \\
\text { rate (\%) }\end{array}$} \\
\hline & & $(-)$ & $(+)$ & $(++)$ & $(+++)$ & & \\
\hline Malignant & 101 & 4 & 12 & 54 & 31 & $96.04 *$ & $84.16^{\#}$ \\
\hline Borderline & II & 3 & 5 & 3 & 0 & 72.72 & 27.27 \\
\hline Benign & 21 & 9 & 9 & 2 & I & 57.14 & 14.29 \\
\hline Normal & 15 & 12 & 3 & 0 & 0 & 20 & 0 \\
\hline
\end{tabular}

Notes: *Compared with the positive rate of borderline, benign, and normal groups, PRDXI in the malignant group was higher (all $P<0.01$ ). "Compared with the high positive rate of borderline, benign, and normal groups, PRDXI in the malignant group was higher (all $P<0.05$ ).

Abbreviation: PRDXI, peroxiredoxin I.

\section{PRDXI protein level can indicate prognosis in ovarian cancer}

The 101 cases of malignant epithelial ovarian tumors were divided into two groups, PRDX1 high $(++/++)$ and low expression groups $(-/+)$, on the basis of the IHC results. Statistical analysis revealed that high PRDX1 expression was significantly correlated with increased tumor FIGO stage, lymph node metastasis, and poor differentiation (all $P<0.05$; Table 2). In order to visualize the results, we mapped the box plot of correlation of PRDX1 expression with clinicopathologic parameters (Figure 2). A significant statistical difference was not noted between serous and non-serous subtypes of cancer regarding the expression of PRDX1 (all $P>0.05$; Table 2).

These patients were followed up while contact was lost with 18 patients. The remaining 83 patients were followed up until September 30, 2017 (follow-up duration: from 1 to 98 months). Thirty-two patients died during follow-up (38.55\%).
Cox regression analysis was used to analyze the impact of the clinical and pathological parameters on the prognosis of ovarian cancer patients. Univariate analysis demonstrated that FIGO stage $(\mathrm{HR}=6.775,95 \% \mathrm{CI}=2.061-22.270$, $P=0.002)$, PRDX1 expression level (HR=7.154, 95\% $\mathrm{CI}=2.171-23.570, P=0.001)$, and lymph node metastasis (HR=2.56, 95\% $\mathrm{CI}=1.202-5.452, P=0.015)$ significantly correlated with patients' overall survival (Table 3). Forest map was used to visualize the univariate and multivariate Cox regression analyses (Figure 3). Data were analyzed by $\mathrm{KM}$ analysis and the result was tested by the log-rank test. The 5-year survival time in the PRDX1 high expression group was significantly lower than that of the PRDX1 low expression group (Figure 4A). Stage III-IV patients presented with lower survival time than stage I-II patients (Figure 4B). Patients with lymph node metastasis presented with lower survival time than patients without metastasis (Figure 4C). Multivariate analysis demonstrated that high expression of PRDX1 (HR=10.033, 95\% CI=1.956-51.462, $P=0.006)$, advanced clinical stage $(\mathrm{HR}=4.569,95 \%$ $\mathrm{CI}=1.226-17.031, P=0.024)$, and poor differentiation (HR=3.086, 95\% CI=1.012-9.409, $P=0.048)$ were independent risk factors for the prognoses of ovarian cancer patients (Table 3).

\section{Overall survival and expression differences of PRDXI in different databases}

We found that PRDX1 expression did not have significant differences in the overall survival in TCGA database

Table 2 Relationship between PRDXI expression and clinicopathologic parameters for malignant ovarian serous tumors

\begin{tabular}{|c|c|c|c|c|c|c|c|}
\hline \multirow[t]{2}{*}{ Item } & \multirow[t]{2}{*}{$\mathbf{n}$} & \multicolumn{2}{|c|}{ Low } & \multicolumn{2}{|l|}{ High } & \multirow{2}{*}{$\begin{array}{l}\text { High positive } \\
\text { rate }(\%)\end{array}$} & \multirow[t]{2}{*}{$P$-value } \\
\hline & & $(-)$ & $(+)$ & $(++)$ & $(+++)$ & & \\
\hline FIGO stage & & & & & & & $<0.05$ \\
\hline I-II & 39 & 4 & 8 & 17 & 10 & 69.23 & \\
\hline III-IV & 62 & I & 4 & 37 & 20 & 91.94 & \\
\hline \multicolumn{8}{|l|}{ Differentiation } \\
\hline Well-moderate & 44 & 3 & 11 & 21 & 9 & 55.00 & $<0.05$ \\
\hline Poor & 57 & 2 & 4 & 33 & 18 & 89.47 & \\
\hline Lymph node metastasis & & & & & & & $<0.05$ \\
\hline Yes & 29 & 0 & 3 & 18 & 8 & 89.66 & \\
\hline No & 65 & 4 & 16 & 26 & 19 & 69.23 & \\
\hline Unknown* & 7 & 0 & 1 & 6 & 0 & 85.71 & \\
\hline Pathological subtype & & & & & & & $>0.05$ \\
\hline Serous & 55 & 2 & 6 & 29 & 18 & 85.45 & \\
\hline Mucinous & 4 & 0 & 1 & I & 2 & 75.00 & \\
\hline Endometrioid & 14 & 0 & 3 & 7 & 4 & 70.57 & \\
\hline Clear cell carcinoma & 8 & I & 0 & 5 & 2 & 87.50 & \\
\hline Poorly differentiated adenocarcinoma & 20 & I & 2 & 12 & 5 & 85.00 & \\
\hline
\end{tabular}

Note: *Patients without lymphadenectomy.

Abbreviations: FIGO, International Federation of Gynecology and Obstetrics; PRDXI, peroxiredoxin I. 
A

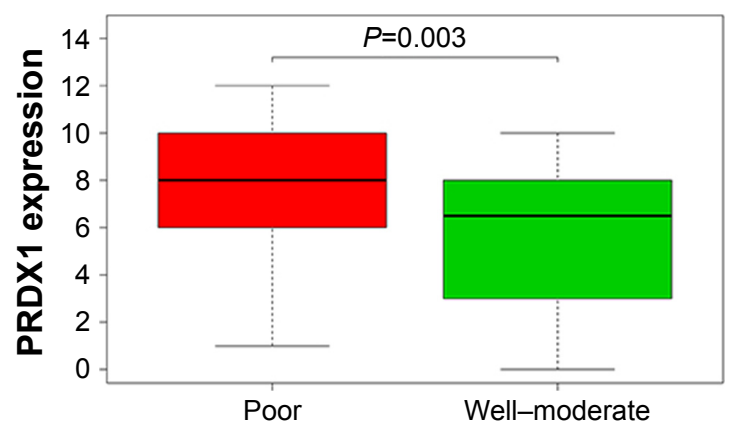

B

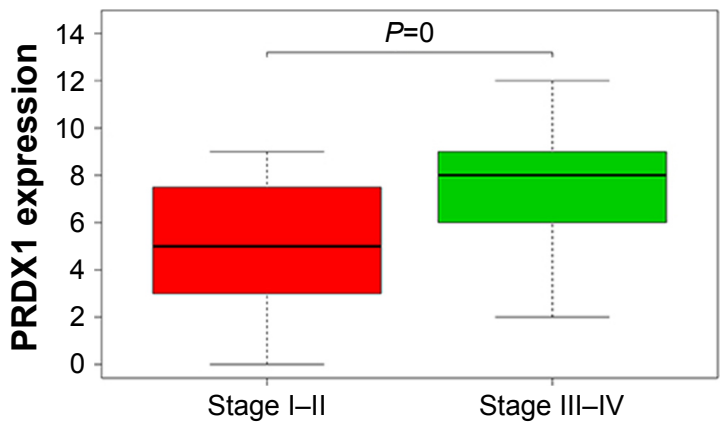

C

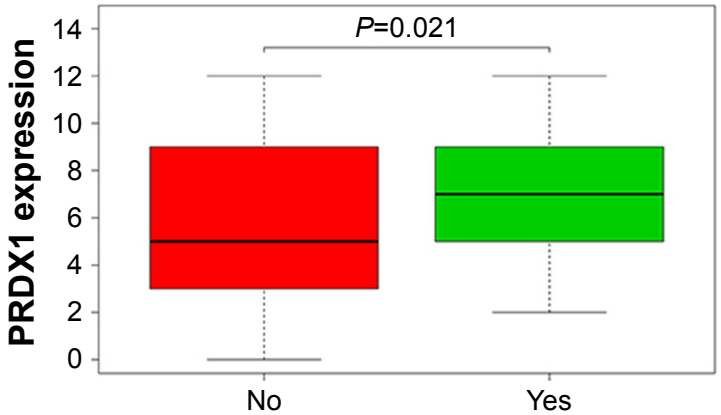

Figure 2 Relationship between PRDXI expression and clinicopathologic parameters.

Note: Correlation of PRDXI expression with differentiation (A), stage (B), and lymph node metastasis (C).

Abbreviation: PRDXI, peroxiredoxin I.

containing 424 patients (Figure 5B). In the KM plotter database, the screening criteria are as follows: 1) "Cancer, Ovarian Cancer;" 2) "Gene: PRDX1;" 3) "Survival: OS;" 4) "Follow-up threshold: 60 months;" 5) "Histology: Serous $(\mathrm{n}=1,232)$ " Information "Use earlier release of the database: 2017 version;" Allowance "Array quality control: exclude outlier arrays." Finally, 1,156 cases were included, with 842 patients demonstrating high PRDX1 expression and 314 patients low expression. The KM survival curve showed a significant correlation between the expression level of PRDX1 and prognosis (Figure 5A), log-rank $P=0.035$, $\mathrm{HR}=1.23$ (1.01-1.48), that is, the higher the PRDX1 expression level, the worse the prognosis. We found that PRDX1 was highly expressed in liver cancer, stomach cancer, lymphoma, bladder cancer, etc through the Oncomine database analysis and was also overexpressed in eight ovarian cancer data sets (Figure 5C and D). However, we did not have access to extract the survival data of PRDX1 in the Oncomine database.

\section{Correlation of PRDXI expression with copy number variation and methylation data}

To investigate the high expression of PRDX1 in ovarian cancer, we performed a correlation analysis of copy number and methylation level. There were $>100$ samples that

Table 3 Cox regression analysis of overall survival of ovarian epithelial serous tumors

\begin{tabular}{|c|c|c|c|c|c|c|}
\hline \multirow[t]{2}{*}{ Variables } & \multicolumn{3}{|c|}{ Univariate analysis } & \multicolumn{3}{|c|}{ Multivariate analysis } \\
\hline & HR & $95 \% \mathrm{Cl}$ of $\mathrm{HR}$ & $P$-value & HR & $95 \% \mathrm{Cl}$ of $\mathrm{HR}$ & $P$-value \\
\hline Age ( $<60$ vs $\geq 60$ years) & 1.454 & $0.721-2.932$ & 0.296 & 0.612 & $0.223-1.684$ & 0.342 \\
\hline FIGO stage (I-II vs III-IV) & 6.775 & $2.06 I-22.270$ & $0.002^{*}$ & 4.569 & $1.226-17.031$ & $0.024^{*}$ \\
\hline PRDXI expression (low vs high) & 7.154 & $2.17 \mid-23.570$ & $0.00 I^{*}$ & 10.033 & $1.956-51.462$ & $0.006 *$ \\
\hline Lymph node metastasis (yes vs no) & 2.56 & $1.202-5.452$ & $0.015^{*}$ & 0.737 & $0.294-1.845$ & 0.514 \\
\hline Differentiation (poor vs well-moderate) & 1.648 & $0.760-3.572$ & 0.206 & 3.086 & $1.012-9.409$ & $0.048 *$ \\
\hline Pathological subtype (serous vs nonserous) & 1.230 & $0.604-2.502$ & 0.568 & 2.787 & $0.794-9.783$ & 0.110 \\
\hline
\end{tabular}

Note: $* P<0.05$.

Abbreviations: FIGO, International Federation of Gynecology and Obstetrics; PRDXI, peroxiredoxin I. 
A

Univariate analysis

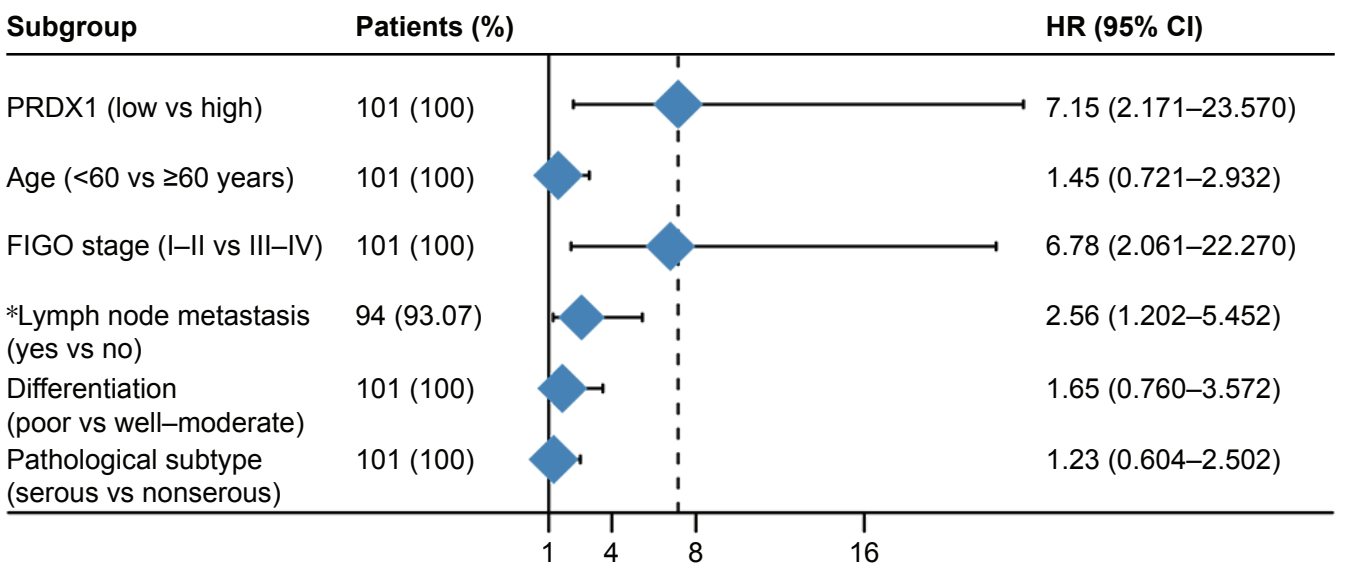

B

Multivariate analysis

\begin{tabular}{|c|c|c|}
\hline Subgroup & Patients (\%) & HR $(95 \% \mathrm{Cl})$ \\
\hline PRDX1 (low vs high) & $101(100)$ & 10.03 (1.956-51.462) \\
\hline Age (<60 vs $\geq 60$ years) & $101(100)$ & $0.61(0.223-1.684)$ \\
\hline FIGO stage (I-II vs III-IV) & $101(100)$ & 4.57 (1.226-17.031) \\
\hline $\begin{array}{l}\text { *Lymph node metastasis } \\
\text { (yes vs no) }\end{array}$ & 94 (93.07) & $0.74(0.294-1.845)$ \\
\hline $\begin{array}{l}\text { Differentiation } \\
\text { (poor vs well-moderate) }\end{array}$ & $101(100)$ & $3.09(1.012-9.409)$ \\
\hline $\begin{array}{l}\text { Pathological subtype } \\
\text { (serous vs nonserous) }\end{array}$ & $101(100)$ & $2.79(0.794-9.783)$ \\
\hline
\end{tabular}

Figure 3 Forest map based on univariate $(\mathbf{A})$ and multivariate $(\mathbf{B})$ Cox regression analyses.

Note: $* P<0.05$

Abbreviations: FIGO, International Federation of Gynecology and Obstetrics; PRDXI, peroxiredoxin I.

underwent copy number amplification in TCGA ovarian cancer data. Correspondingly, the expression of the samples with copy number amplification also increased, indicating that high expression of PRDX1 in ovarian cancer was partly caused by copy number amplification (Figure 6A). PRDX1 expression and methylation level correlation analysis showed a negative correlation, and the hypomethylation level of PRDX1 resulted in high expression of PRDX1 (Figure 6B).

\section{Inhibition of PRDXI expression downregulated ovarian cancer cell proliferation, migration, and metastasis}

Knockdown of PRDX1 expression was performed using siRNA. Transfection efficiency was measured with Western blot analyses (Figure 7A). We found that siRNA1 interference to be the most efficient; so it was selected for subsequent biological behavioral experiments. Flow cytometry revealed that the number of G1 phase cells in the transfection group was significantly increased, and the number of $S$ phase cells was decreased (Figure 7B), indicating that G1/S phase retardation had occurred. A wound healing assay indicated that PRDX1 knockdown could decrease the migration ability of Caov-3 cells (Figure 7C). Transwell assays showed that the migration and invasion abilities of PRDX1-siRNAtransfected groups were significantly inhibited (Figure 7D). PRDX1 knockdown markedly decreased the proliferation of Caov-3 cells (Figure 7E).

\section{PRDXI influences key proteins to regulate $W n t$ signaling pathway}

To investigate further the pathways that PRDX1 may regulate, gene enrichment analysis of PRDX1 was performed. The results indicated that Wnt signaling pathways-related gene clusters were enriched in PRDX1 high expression samples (Figure 8A). Pathways related to "oxidative phosphorylation" and "pathways in cancer" were also identified as 

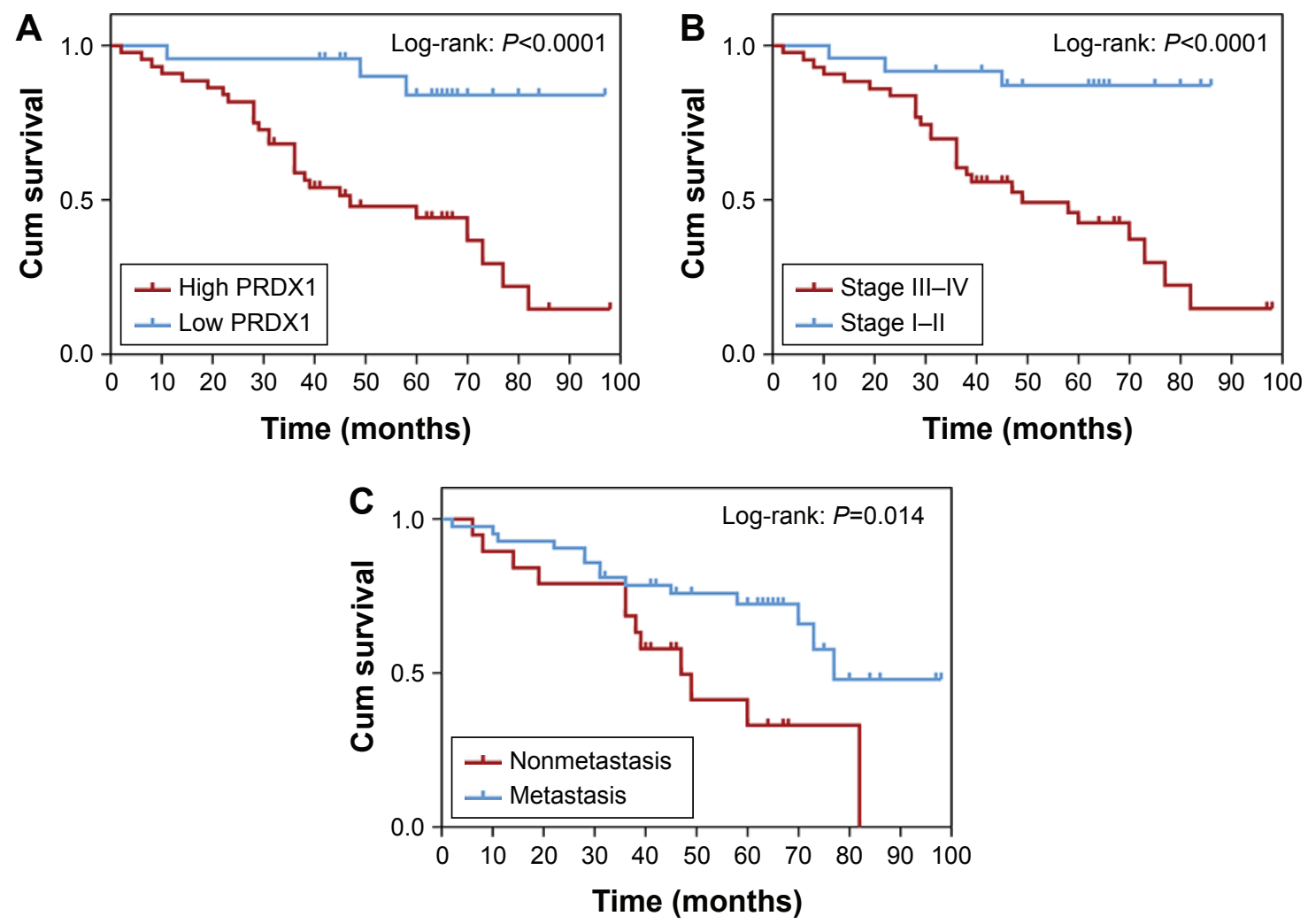

Figure 4 Kaplan-Meier curves for overall survival of patients with epithelial ovarian cancer.

Note: Correlation of PRDXI expression (A), clinical stage (B), and lymph node metastasis (C) with overall survival.

Abbreviations: Cum, cumulative; PRDXI, peroxiredoxin I.

significantly altered along with aberrant PRDX1 expression (Figure 8B and C).

The protein expression levels of $\beta$-catenin in the Wnt pathway was obviously downregulated after the siRNA transfection, as detected by Western blot. It has been reported that Wnt signaling pathways participate in the process of epithelialmesenchymal transition (EMT) and regulate the occurrence and development of cancer. We then examined the expression levels of EMT-related proteins. We found that the expression of vimentin was significantly downregulated, E-cadherin protein expression was significantly upregulated (Figure 8D and E), and the cell morphology changed from spindle to round after transfection (Figure 8F), indicating that inhibition of PRDX1 expression can inhibit the occurrence of EMT.

\section{Function and enrichment analysis}

One hundred and one genes associated with PRDX1 were obtained from the web site of the Gene-Cloud of Biotechnology Information (GCBI, https://www.gcbi.com. cn/gclib/html/index) (supplementary table). Based on the DAVID database, biological functions and pathways for those genes were analyzed. The first 30 Gene Ontology (GO) terms of biological function and pathways were visualized by a "ggplot2" R package (Hadley Wickham, New Zealand). It was found that genes were mainly enriched in PI3K/Akt signaling pathway, p53 signaling pathway, pathways in cancer, FoxO signaling pathway, ubiquitin-mediated proteolysis, cell cycle, and Wnt signaling pathway (Figure 9A). It was also found that the genes were mainly involved in the regulation of G1/S transition of mitotic cell cycle, negative regulation of apoptotic process, positive regulation of cell proliferation, and cell cycle arrest biological function (Figure 9B). We have proved that inhibiting the expression of PRDX1 can suppress G1/S phase transition (Figure 7B). Bioinformatics analysis further validates our experimental results.

\section{Construction of protein-protein interaction network and module screening} There were 101 nodes and 541 edges in the protein-protein interaction network (Figure 10). The average clustering coefficient was $0.487 ; P<1.0 \mathrm{e}-16$. MCODE1 network screened out from the protein-protein interaction network (Figure 11A). The pathway enrichment of genes in MCODE1 were mainly enriched in cell cycle, $\mathrm{PI} 3 \mathrm{~K} /$ Akt signaling pathways, FoxO signaling pathway, and biological process such as GO:0000086 G2/M transition 
A

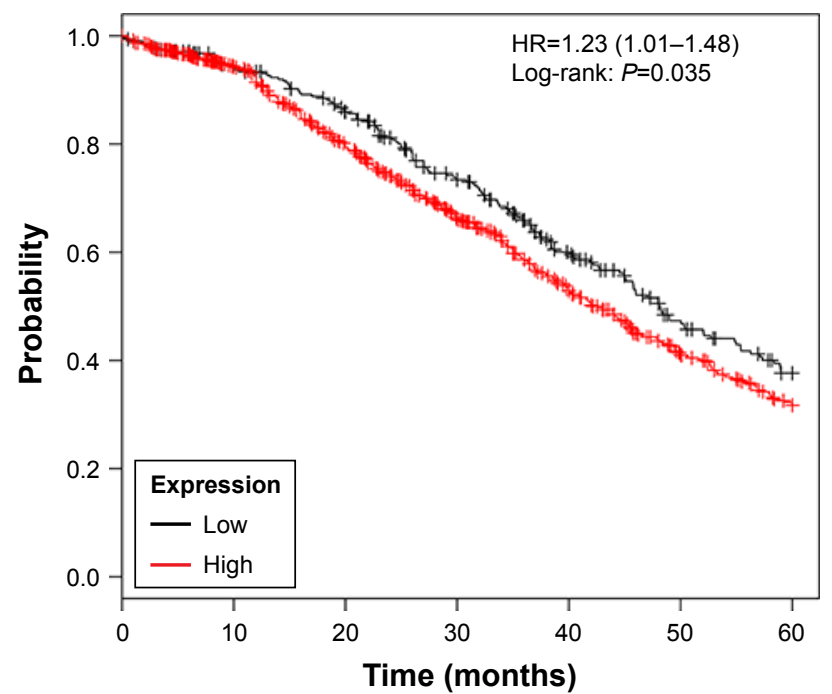

Number at risk

$\begin{array}{llllllll}\text { Low } & 314 & 275 & 239 & 185 & 128 & 88 & 62 \\ \text { High } & 842 & 699 & 550 & 394 & 269 & 168 & 113\end{array}$

\section{C}

\begin{tabular}{|c|c|c|c|c|c|c|c|c|c|c|}
\hline \multicolumn{11}{|c|}{$\begin{array}{c}\text { Comparison of PRDX1 across } 8 \text { analyses } \\
\text { Overexpression/copy number gain }\end{array}$} \\
\hline Median rank & $P$-value & Gene & & & & & & & & \\
\hline \multirow{2}{*}{$1,268.5$} & \multirow{2}{*}{0.037} & \multirow{2}{*}{ PRDX1 } & & & & & & & & \\
\hline & & & 1 & 2 & 3 & 4 & 5 & 6 & & 8 \\
\hline
\end{tabular}

Legend

$\begin{array}{lc}\text { 1. Cancer type: ovarian cancer } & \text { 5. Ovarian cancer type: overian carcinoma } \\ \text { Barretina CellLine, Nature, 2012 } & \text { Etemadmoghadam Ovarian, Clin Cancer } \\ \text { 2. Cancer type: ovarian cancer } & \text { Res, 2009 } \\ \text { Barretina Cellline 2, Nature, 2012 } & \text { 6. Cancer type: ovarian cancer } \\ \text { 3. Ovarian adenocarcinoma type: } & \text { Garnett CellLine, Nature, 2012 } \\ \text { ovarian clear cell adenocarcinoma } & \text { 7. Ovarian adenocarcinoma type: ovarian } \\ \text { Bittner Ovarian, Not Published, 2005 } & \text { endometrioid adenocarcinoma } \\ \text { 4. Ovarian adenocarcinoma type: } & \text { Lu Ovarian, Clin Cancer Res, 2004 } \\ \text { ovarian serous adenocarcinoma } & \text { 8. Ovarian adenocarcinoma type epithelia: } \\ \text { Etemadmoghadam Ovarian, Clin Cancer } & \text { ovarian serous adenocarcinoma } \\ \text { Res, 2009 } & \text { Ramakrishna Ovarian, PLoS One, 2010 }\end{array}$

\section{B \\ Overall survival}

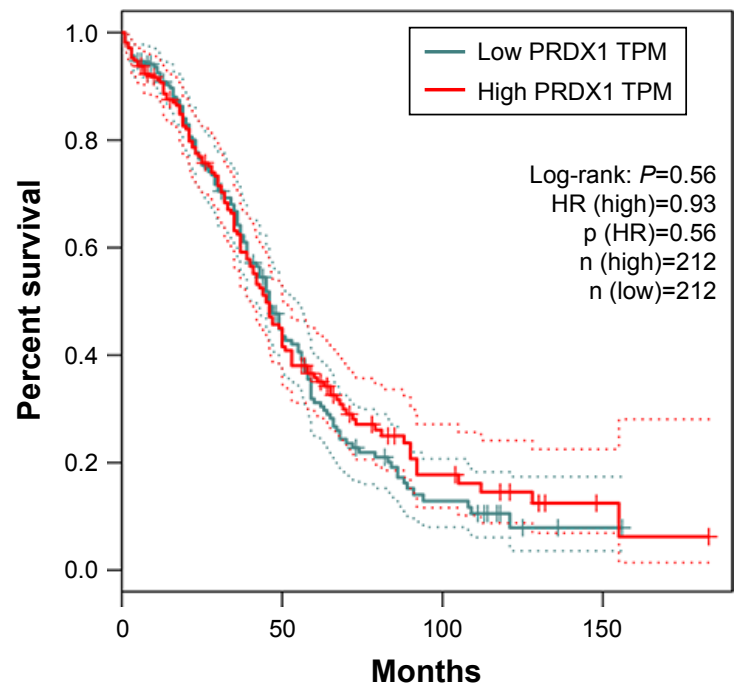

D Comparison of PRDX1 across 11 analyses Overexpression/copy number gain

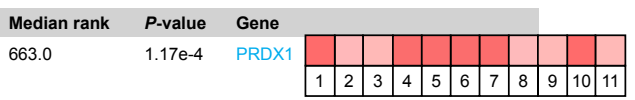

Legend

\begin{tabular}{ll}
\hline 1. Cancer type: bladder cancer & 7. Cancer type: esophageal cancer \\
Barretina CellLine, Nature, 2012 & Garnett CellLine, Nature, 2012 \\
2. Cancer type: breast cancer & 8. Cancer type: gastric cancer \\
Barretina CellLine, Nature, 2012 & Jaiswal Multi-cancer, Cancer Cell, 2010 \\
3. Cancer type: liver cancer & 9. Cancer type: colorectal cancer \\
Barretina CellLine, Nature, 2012 & Staunton CellLine, Proc Natl Acad Sci \\
4. Cancer type: ovarian cancer & U S A, 2001 \\
Barretina CellLine, Nature, 2012 & 10. Cancer type: kidney cancer \\
5. Cancer type: sarcoma & Staunton CellLine, Proc Natl Acad Sci \\
Barretina CellLine 2, Nature, 2012 & U S A, 2001 \\
6. Cancer type: lung cancer & 11. Cancer type: lymphoma \\
Compendia CellLine, Not Published, 2007 & Su Normal, Proc Natl Acad Sci \\
& U S A, 2002
\end{tabular}

Figure 5 Overall survival and expression differences of PRDXI in different databases.

Notes: KM curves for overall survival of patients in KM plotter (A) and TCGA (B). Differential expression of PRDXI in eight ovarian cancer data sets (C) and II cancer data sets (D) from Oncomine.

Abbreviations: PRDXI, peroxiredoxin I; KM, Kaplan-Meier; TCGA, the Cancer Genome Atlas; TPM, transcripts per million reads.
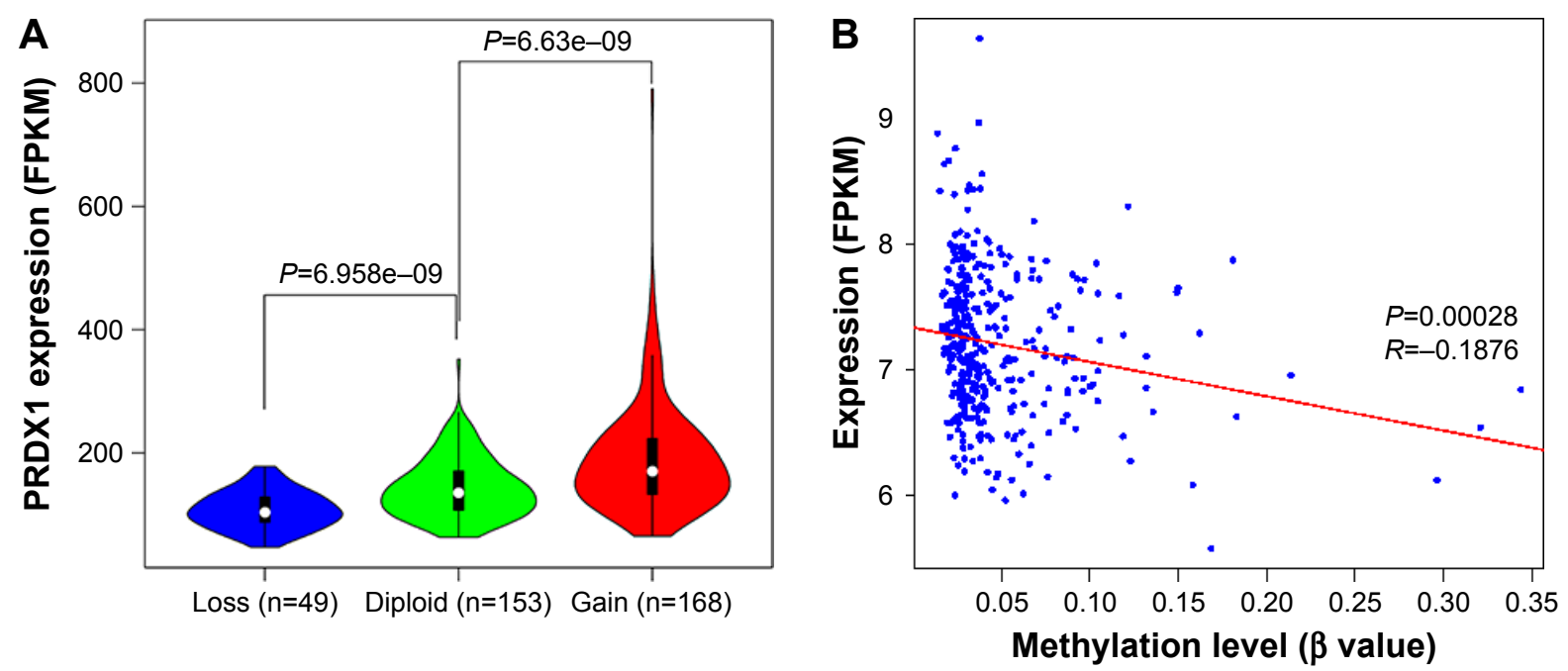

Figure 6 Correlation analysis of PRDXI expression with copy number variation (A) and methylation data (B).

Abbreviations: PRDXI, peroxiredoxin I; FPKM, fragments per kilobase of transcript per million mapped reads. 
A

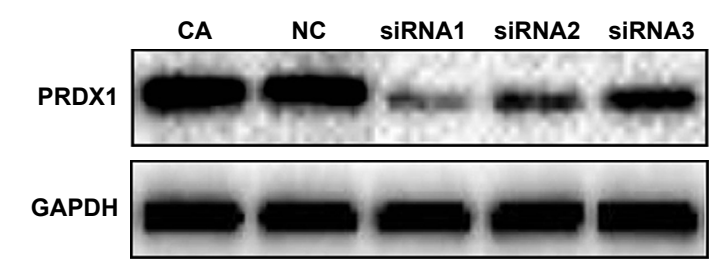

B

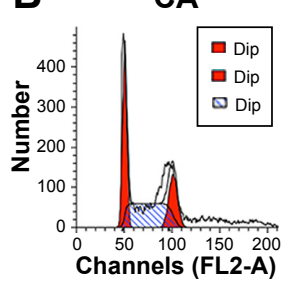

NC

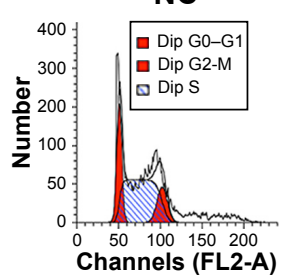

SiRNA

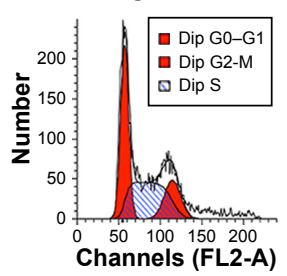

C

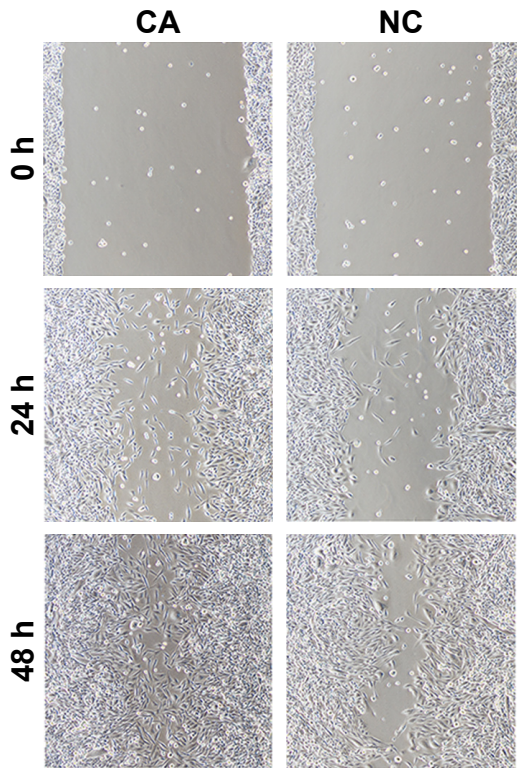

SiRNA

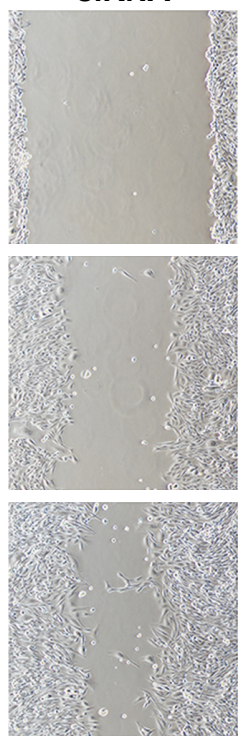

D CA

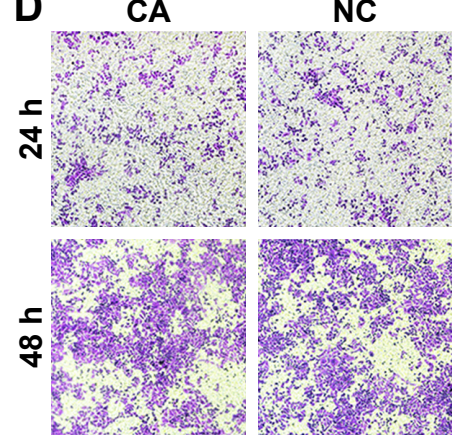

E

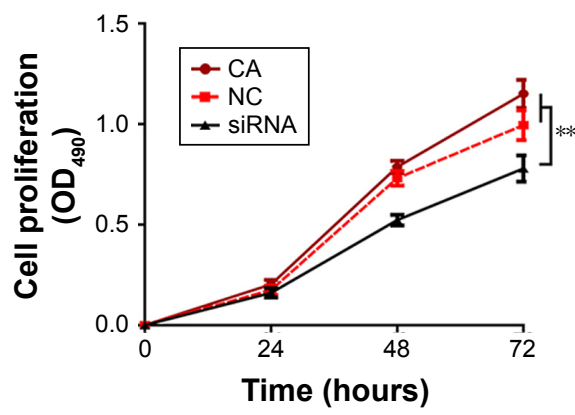

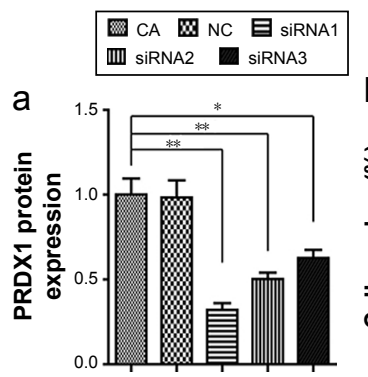
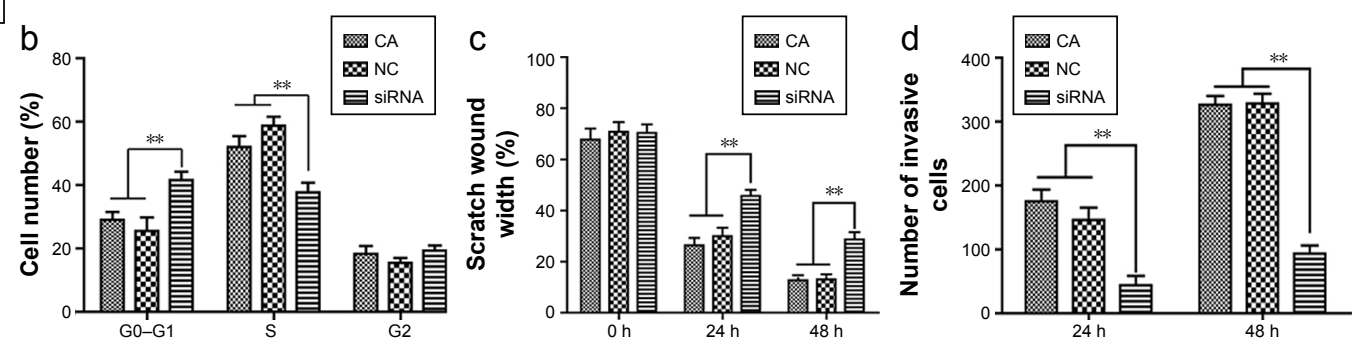

Figure 7 Decreased PRDXI inhibited proliferation, invasion, and metastasis capacities of ovarian cancer cells in vitro.

Notes: The expression of PRDXI after PRDXI knockdown by different siRNA was evaluated by immunoblot staining in ovarian cancer cell lines Caov-3 (A). Cell cycle of transfected cells was studied using flow cytometry (B). Invasion of PRDXI knockdown ovarian cancer cells was measured by wound healing (C). Migration of PRDXI knockdown cells was measured by transwell assay (D). The proliferating capability of transfected cells was evaluated using MTT (E). Quantification of the immunoblot staining results (a), flow cytometry results (b), wound healing assay results (c), and transwell invasion assay results (d). siRNAI, siRNA2, and siRNA3 denote PRDXI-siRNA transfection. NC indicates an empty-plasmid transfected cell line and $C A$ is an untreated cell line. ${ }^{*} P<0.05, * * P<0.01$.

Abbreviations: PRDXI, peroxiredoxin I; siRNA, small interfering RNA.

of mitotic cell cycle, GO:0006605 protein targeting, GO:0042059 negative regulation of EGFR signaling pathway, and GO:0050900 leukocyte migration were involved (Figure 11B and C). A flowchart of the functional enrichment analysis of PRDX1-related genes and construction of protein interaction network is shown in Figure 12.

\section{Discussion}

The mortality rate caused by ovarian cancer is the highest of all gynecological tumors, with $70 \%$ of cases diagnosed at an advanced stage. Invasion and metastasis are the most common causes for the high mortality. So, it is very important to explore the molecular mechanisms involved in the development of ovarian cancer and to identify effective tumor biomarkers. PRDX1 is a family of antioxidant enzymes that protects cells from oxidative damage by active oxygen. Although previous studies have shown abnormal expression of PRDX1 in ovarian cancer cells, its detailed functions and regulatory mechanisms were not fully understood. Therefore, 

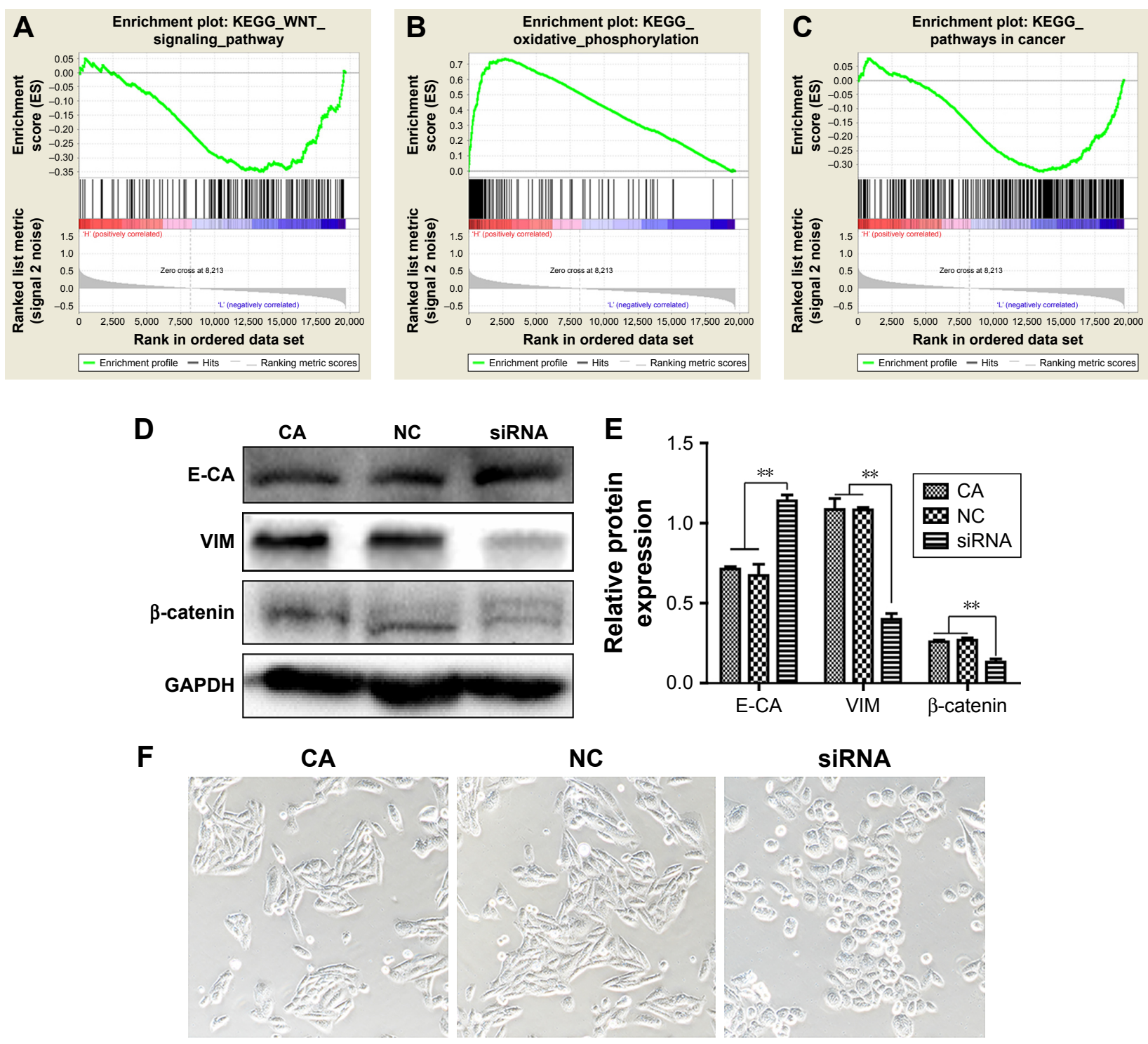

Figure 8 PRDXI correlated enrichment gene analysis.

Notes: (A) Wnt signaling pathway $(P=0.0028$; $F D R=0.077$; enrichment score $=-0.349)$. (B) Oxidative phosphorylation $(P=0.000$; $F D R=0.000$; enrichment score=0.739). (C) Pathways in cancer ( $P=0$; FDR=0.058; enrichment score=-0.325). (D) Immunoblot staining results showed the expression levels of $E-C A$, VIM, $\beta$-catenin after gene transfection. (E) Quantification of the immunoblot staining results. (F) The change of cell morphology, before and after PRDXI gene transfection. NC indicates an emptyplasmid transfected cell line and $C A$ is an untreated cell line. $* * P<0.05$.

Abbreviations: PRDXI, peroxiredoxin I; FDR, false discovery rate; siRNA, small interfering RNA; E-CA, E-cadherin; VIM, vimentin; KEGG, Kyoto Encyclopedia of Genes and Genomes.

it was necessary to thoroughly research the mechanism of action of PRDX1 in ovarian cancer.

Using IHC, we found that the expression of PRDX1 in ovarian tumor tissues was markedly higher than that in borderline and benign epithelial ovarian tumor or normal ovarian tissues (all $P<0.05$ ). The expression of PRDX1 was correlated with specific ovarian cancer clinicopathologic features, such as advanced clinical stage, lymph node metastasis, and poor tissue differentiation (all $P<0.05$ ), suggesting that PRDX1 overexpression can increase the malignancy of ovarian cancer. The expression level of PRDX1, clinical stage, and lymph node metastasis were found to correlate with prognosis (all $P<0.05$ ) by univariate Cox regression analysis. In comparison, Cox multivariate regression analysis indicated that advanced clinical stage, low tissue differentiation, and high expression of PRDX1 were independent risk factors affecting the prognosis of epithelial ovarian cancer $(P<0.05)$. Thus, the expression level of PRDX1 may become an indicator useful in evaluating the prognosis of patients with epithelial ovarian cancer.

So far, only one study has demonstrated that PRDX1 was linked to the prognosis of patients with epithelial ovarian cancer. ${ }^{10}$ Chung et al studied 195 specimens of epithelial ovarian tumors by immunohistochemical staining and 
A

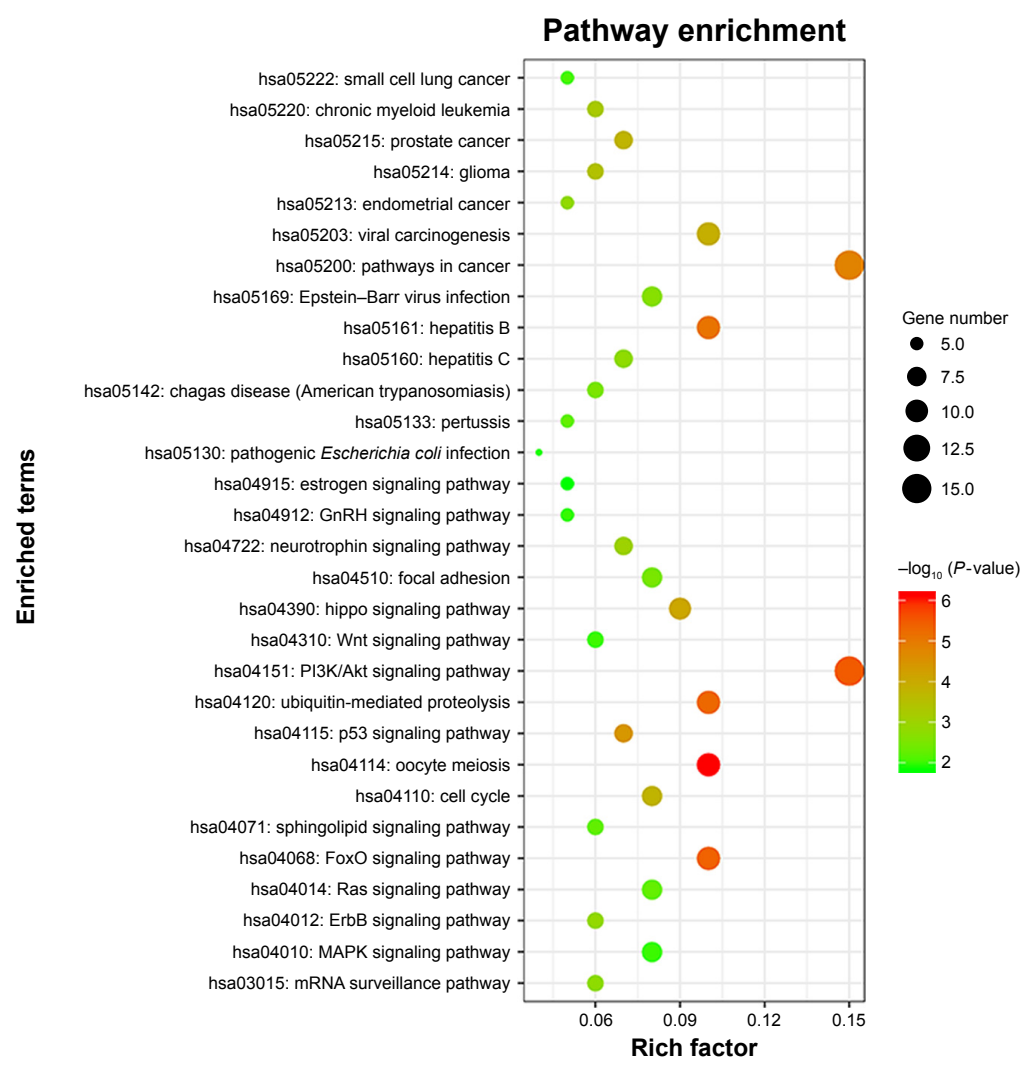

B

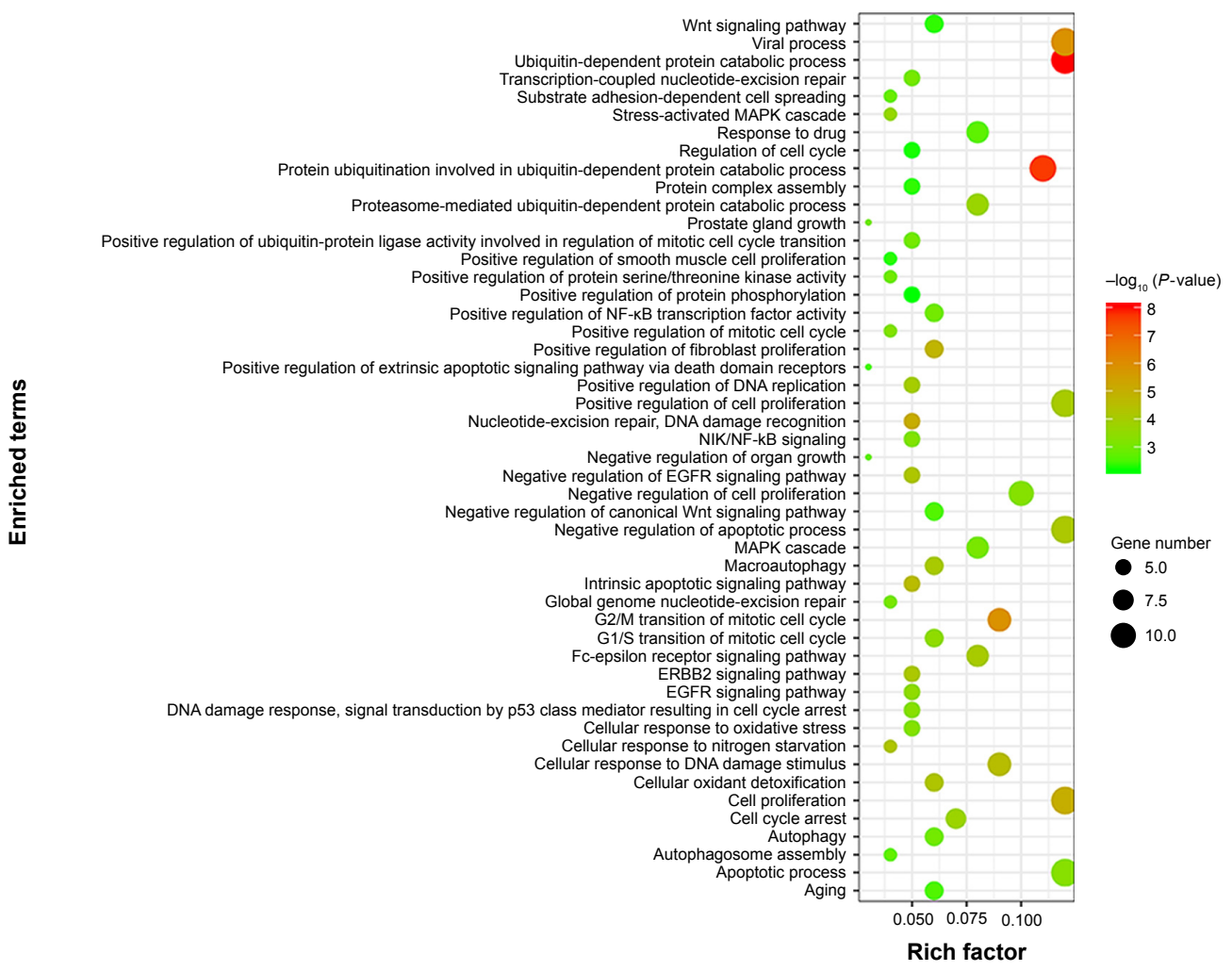

Figure 9 Network of enriched terms and top 30 of GO terms and pathway enrichment analysis.

Notes: (A) Bubble pattern of enriched biological function of GO terms in the genes associated with PRDXI. (B) Bubble pattern of enriched pathway in the genes associated with PRDXI. The $Y$-axis represents the name of the pathway or function, and the $X$-axis represents the percentage of this node in the whole network. The size of the bubble represents the number of genes in this signaling pathway or the number of genes involved in this function. The color of the bubble indicates the $P$-value of enrichment. The darker the color, the more significant the result.

Abbreviations: PRDXI, peroxiredoxin I; GO, Gene Ontology. 


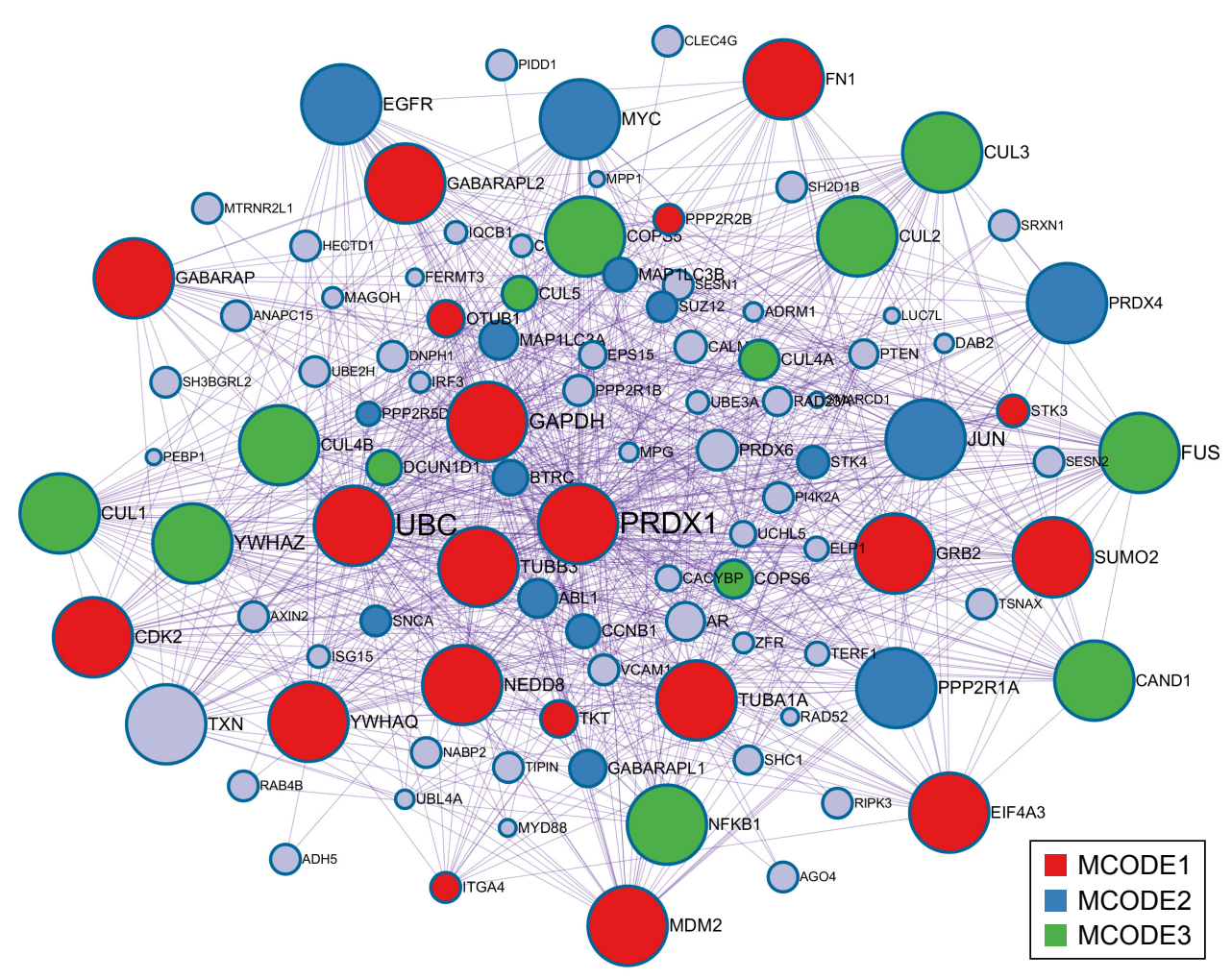

Figure I0 PRDXI protein-protein interaction network.

Notes: Each node represented a protein, and the edge between two nodes represented the relationship between two proteins. Different thicknesses of an edge represented the strength of the interaction. The more interaction the protein had, the bigger the node.

Abbreviations: PRDXI, peroxiredoxin I; MCODE, Molecular Complex Detection.

obtained similar results. The study of Chung et al involved 77 cases of epithelial ovarian cancer, including 43 with serous carcinoma, nine with mucinous adenocarcinoma, 17 with endometrial adenocarcinoma, and eight with clear cell carcinoma. It was found that PRDX1 was highly expressed in ovarian serous carcinoma and was related to patients' prognoses. However, it was found that the expression level of PRDX1 was not related to clinical stage, tissue differentiation, and lymph node metastasis, presumably due to the lack of adequate malignant tumor specimens. In the Hoskins et al study, the interstitial fluid and ascites of four patients with ovarian serous papillary carcinoma were analyzed by proteomic analysis. ${ }^{15}$ Immunoassay analysis was performed on the selected PRDX1. It was found that the expression level of PRDX1 in ovarian serous papillary carcinoma was six times higher than that in normal tissue or benign tumors. This suggested that PRDX1 could be used as a potential diagnostic biomarker for epithelial ovarian cancer.

KM plotter, TCGA, and Oncomine database were combined to verify further the overall survival and expression of PRDX1. However, there was no significant difference between the expression level of PRDX1 and prognosis. Then, more cases were included from the KM plotter database.
We found the expression of PRDX1 was significantly associated with the prognosis of patients, which is consistent with our experimental result. At the same time, we found that PRDX1 was highly expressed in multiple tumors and was overexpressed in eight ovarian cancer data sets through the Oncomine database, further validating our IHC results. In order to explore the reasons for the high expression of PRDX1 in ovarian cancer, a correlation analysis of copy number and methylation level was performed using TCGA database. It suggested that $>100$ samples were amplified by copy number, and the corresponding gene expression was also increased. We speculate that the high expression of PRDX1 in ovarian cancer is partly due to copy number amplification. DNA methylation is one of the important modifications of the genome of eukaryotic cells. It regulates the expression of genes by interacting with transcription factors or by altering chromatin structure, and regulates biological genetic information from epigenetic levels. PRDX1 expression and methylation level correlation analysis showed a negative correlation, and the hypomethylation level of PRDX1 leads to the high expression of PRDX1.

The PRDX family is made up of six members, PRDX1 to PRDX6. PRDX1 and PRDX3 are typical 2-cysteine 

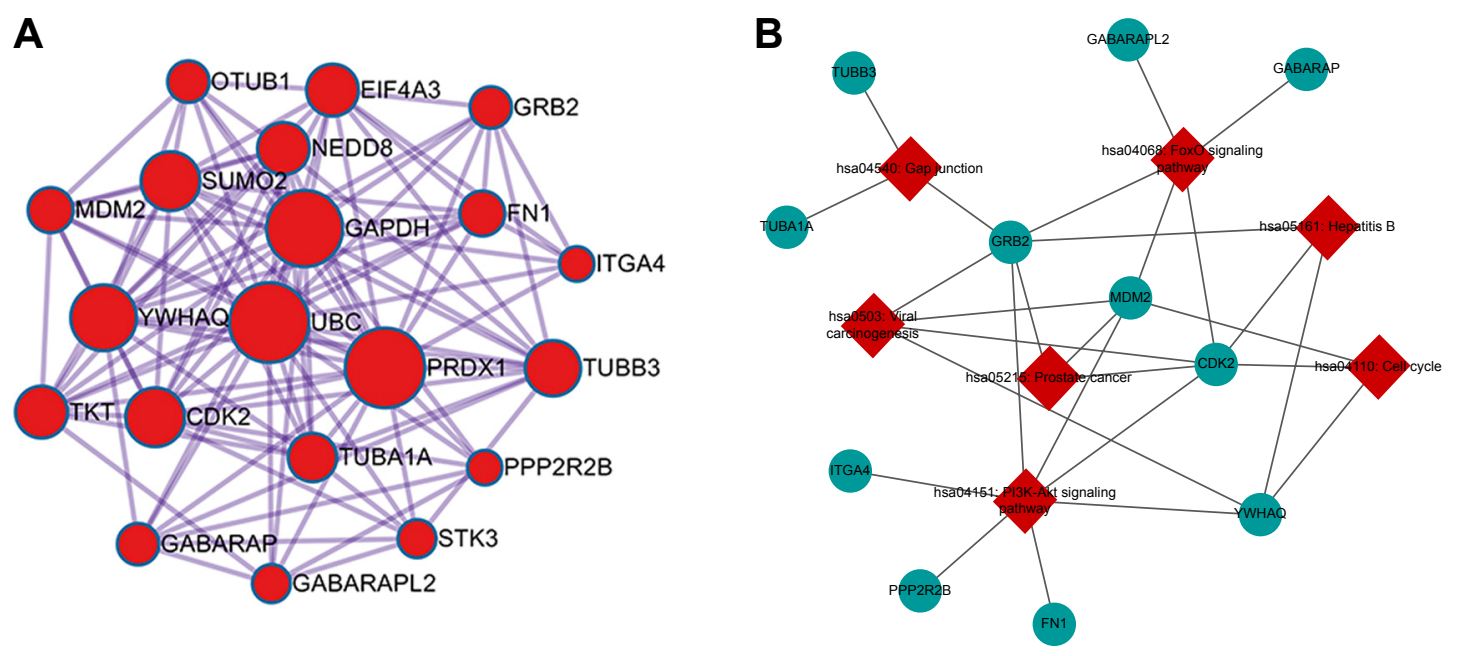

C GO: 1901796 - regulation of signal transduction by p53 class mediator GO: 0070911 - global genome nucleotide-excision repair GO: 0050900 - leukocyte migration GO: 0042059 - negative regulation of EGFR signaling pathway GO: 0038128 - ERBB2 signaling pathway
GO: 0035987 - endodermal cell differentiation
GO: 0034446 - substrate adhesion-dependent cell spreading
GO: 0016236 - macroautophagy
GO: 0007017 - microtubule-based process GO: 0006977 - signal transduction by $\mathrm{p} 53$ class mediator resulting in cell cycle arrest GO: 0006605 - protein targeting GO: 0000086 - G2/M transition of mitotic cell cycle

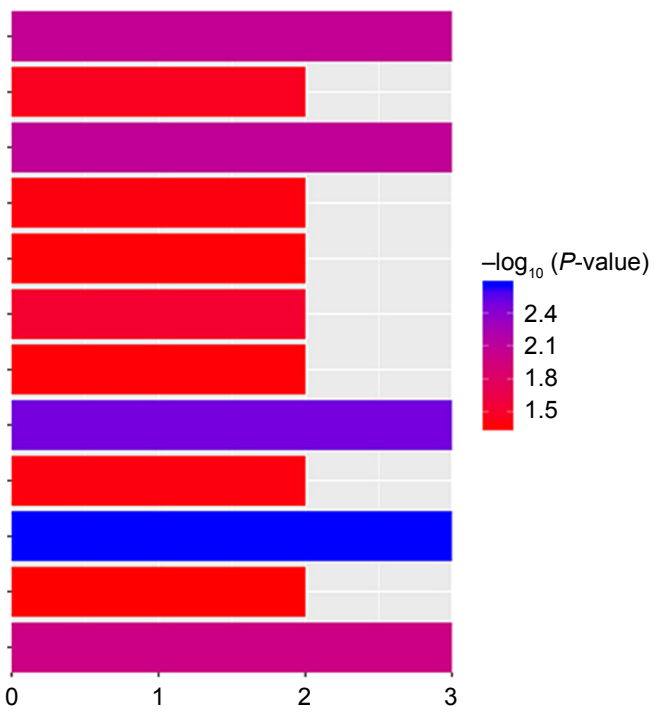

Figure II The MCODE module of PPI and biological function and pathways analysis.

Notes: (A) MCODEI. Each node represented a protein and the edge between two nodes represented the relationship between two proteins. (B) Network of pathways of MCODEI. The circle represents the regulatory gene and the diamond represents the pathway. (C) Bar graph of function enrichment of genes in MCODEI. The Y-axis represents a signaling pathway or function. The $\mathrm{X}$-axis represents the number of genes enriched in this region. The bar color indicates the statistical significance. The bluer the bar, the smaller the $P$-value.

Abbreviations: MCODE, Molecular Complex Detection; GO, Gene Ontology; PPI, protein-protein interaction.

molecules. Duan et al found that the inhibition of PRDX3 expression triggered cisplatin-mediated apoptosis in ovarian cancer cells, which may act through suppression of the NF- $\mathrm{kB}$ signaling pathway. ${ }^{16}$ In a study by Pak et al, overexpression of PRDX6 was demonstrated to reduce the apoptotic effect of cisplatin on human ovarian cancer cells by reducing ROS levels and suppressing the caspase signaling pathway. ${ }^{17}$ A study by He et al showed that PRDX1 knockdown increased ROS accumulation and mitogen-activated protein to enhance lapachone-induced apoptosis. ${ }^{18}$ At present, the relationship between PRDX1 and the mechanisms involved in the development of ovarian cancer has not been investigated. To explore further the influence of PRDX1 on the biological behaviors of ovarian cancer cells, we analyzed the pathways and functions of genes associated with PRDX1 combined with bioinformatics.

In the present study, cell proliferation, invasion, metastasis, and cell cycle were inhibited after knocking down the expression of PRDX1 in Caov-3 ovarian cancer cells. Subsequently, gene enrichment analysis of PRDX1 based on TCGA database was performed to further investigate the molecular mechanisms underlying the effect of PRDX1 on the proliferation, invasion, and metastasis of ovarian cancer cells. We found that the expression of PRDX1 significantly correlated with the Wnt signaling pathways.

PRDX1 is an antioxidant that regulates cell growth, differentiation, apoptosis, and other functions. In the nucleus, PRDX1 affects bioactivities upon gene regulation by 


\section{Proteins that interact with PRDX1 were obtained from} Gene-Cloud of Biotechnology Information

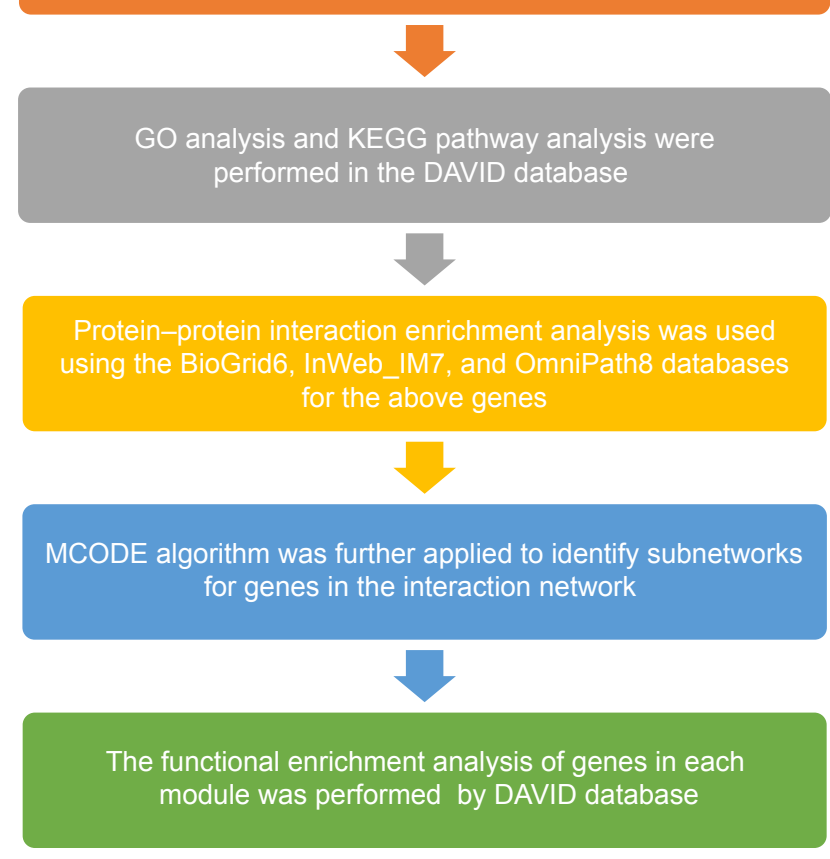

Figure 12 Flowchart of the bioinformatics approach.

Note: Chart of functional enrichment analysis of PRDXI-related genes and construction of protein interaction network.

Abbreviations: PRDXI, peroxiredoxin I; MCODE, Molecular Complex Detection; GO, Gene Ontology; KEGG, Kyoto Encyclopedia of Genes and Genomes.

associating with $\mathrm{p} 53$ or various transcription factors including $\mathrm{c}-\mathrm{Myc}, \mathrm{NF}-\mathrm{KB}$, and AR. Studies also indicate that PRDX1 is involved in $\mathrm{H}_{2} \mathrm{O}_{2}$-induced apoptosis in the nucleus. ${ }^{6}$

It is well known that Wnt signaling pathway participates in the process of EMT and regulates the occurrence and development of cancer, which is mediated by cell proliferation, invasion, and apoptosis. ${ }^{19,20} \mathrm{Ha}$ et al found that knockdown of PRDX1 significantly inhibited TGF- $\beta 1$-induced EMT and cell migration. ${ }^{21}$ We found that the expression levels of $\beta$-catenin in the Wnt pathway were obviously downregulated after shRNA transfection. Meanwhile, the expression of vimentin was significantly downregulated, E-cadherin protein expression was significantly upregulated. These results suggest the potential mechanism of PRDX1 in repressing proliferation and invasion in ovarian cancer by downregulating $\beta$-catenin protein. Thus, we have further demonstrated a relationship between PRDX1 and the development of ovarian cancer.

However, the invasion and metastasis of tumor cells is a complex series of pathophysiological processes, accompanied with a complex regulatory network involving multiple molecules. In this study, genes correlated with PRDX1 were obtained from the GCBI protein database, according to literature-based relationships. Function and pathway enrichment analysis showed that genes were mainly enriched in PI3K/Akt signaling pathway, p53 signaling pathway, pathways in cancer, MAPK signaling pathway, FoxO signaling pathway, ubiquitin-mediated proteolysis, cell cycle, and Wnt signaling pathway. The ubiquitin-proteasome system is the most significant intracellular proteolytic pathway. Target proteins are usually ubiquitinated prior to degradation by the proteasome to participate in the regulation of cell proliferation, apoptosis, and other cell functions. Aberrations in the ubiquitin-proteasome pathway are commonly observed in many cancers. ${ }^{22}$ Moreover, we found that genes in the MCODE module were enriched in cell cycle, PI3K/Akt signaling pathways, FoxO signaling pathway, and biological processes such as G2/M transition of mitotic cell cycle, protein targeting, and negative regulation of EGFR signaling pathway were involved.

The pathways analyzed in the above-mentioned database were verified. Guo et al found that overexpression of miR-510 inhibited PRDX1 expression, thereby activating the PI3K/Akt pathway and promoting breast cancer cell proliferation. ${ }^{23}$ PRDX1 is an upstream effector for JNK and p38 MAPK pathways. ${ }^{24}$ Turner-Ivey et al discovered that inhibition of PRDX1 expression increased ROS-induced $\mathrm{p} 38$ MAPK phosphorylation and promoted $\mathrm{H}_{2} \mathrm{O}_{2}$-induced breast cancer cell apoptosis. ${ }^{25}$ Studies also found that PRDX1 modulated the activity of p38 MAPK, which, in turn, promoted pancreatic cancer invasion. ${ }^{26}$ The biological behaviors of the enriched genes set mainly relate to the positive regulation of cell proliferation, the negative regulation of the apoptotic process, and the regulation of $\mathrm{G} 1 / \mathrm{S}$ transition of the mitotic cell cycle, among others, which is consistent with our present experimental results. Together with our finding, there seems to be enough evidence to speculate that PRDX1 may also affect the biological behaviors of ovarian cancer cells with related genes through the above-mentioned pathways to regulate the development of ovarian cancer. Further investigations are needed to focus on the roles of these potential related genes to elucidate the underlying basic mechanisms of PRDX1 in ovarian cancer.

Collectively, we confirmed that PRDX1 is an independent risk factor for the prognosis of ovarian cancer. Downregulated expression of PRDX1 can suppress ovarian cancer cell proliferation, invasion, and metastasis. Pathways analysis of the interaction network showed that tumor-related signaling pathways such as Wnt, FoxO, cell cycle, and PI3K/Akt and MAPK pathways may be involved. At present, although 
several PRDX1-related studies of cancer are available, we were the first to demonstrate that the inhibition of PRDX1 expression can suppress malignant biological behaviors such as proliferation, invasion, and metastasis of ovarian cancer cells in ovarian cancer. In this study, bioinformatics analysis was innovatively combined to explore PRDX1-related mechanisms involved in the occurrence and development of ovarian cancer, thus providing a new direction for the further study of PRDX1.

\section{Acknowledgments}

This work was supported by grants from the National Natural Science Foundation of China (81472437, 81672590, $81602438,81602304)$ and Shengjing Freedom researchers' plan (201303). The funding body had no role in the design or conduct of the study.

\section{Disclosure}

The authors report no conflicts of interest in this work.

\section{References}

1. Cortez AJ, Tudrej P, Kujawa KA, Lisowska KM. Advances in ovarian cancer therapy. Cancer Chemother Pharmacol. 2018;81(1):17-38.

2. Colombo PE, Fabbro M, Theillet C, Bibeau F, Rouanet P, Ray-Coquard I. Sensitivity and resistance to treatment in the primary management of epithelial ovarian cancer. Crit Rev Oncol Hematol. 2014;89(2):207-216.

3. Rhee SG, Chae HZ, Kim K. Peroxiredoxins: a historical overview and speculative preview of novel mechanisms and emerging concepts in cell signaling. Free Radic Biol Med. 2005;38(12):1543-1552.

4. Rhee SG, Kang SW, Chang TS, Jeong W, Kim K. Peroxiredoxin, a novel family of peroxidases. IUBMB Life. 2001;52(1-2):35-41.

5. Neumann CA, Cao J, Manevich Y. Peroxiredoxin 1 and its role in cell signaling. Cell Cycle. 2009;8(24):4072-4078.

6. Ding C, Fan X, Wu G. Peroxiredoxin 1 - an antioxidant enzyme in cancer. J Cell Mol Med. 2017;21(1):193-202.

7. Ren P, Ye H, Dai L, et al. Peroxiredoxin 1 is a tumor-associated antigen in esophageal squamous cell carcinoma. Oncol Rep. 2013;30(5): 2297-2303.

8. Chen MF, Keng PC, Shau H, et al. Inhibition of lung tumor growth and augmentation of radiosensitivity by decreasing peroxiredoxin I expression. Int J Radiat Oncol Biol Phys. 2006;64(2):581-591.

9. Wang X, He S, Sun JM, Delcuve GP, Davie JR. Selective association of peroxiredoxin 1 with genomic DNA and COX-2 upstream promoter elements in estrogen receptor negative breast cancer cells. Mol Biol Cell. 2010;21(17):2987-2995.
10. Chung KH, Lee DH, Kim Y, et al. Proteomic identification of overexpressed PRDX 1 and its clinical implications in ovarian carcinoma. J Proteome Res. 2010;9(1):451-457.

11. Jalali BM, Bogacki M, Dietrich M, Likszo P, Wasielak M. Proteomic analysis of porcine endometrial tissue during peri-implantation period reveals altered protein abundance. J Proteomics. 2015;125:76-88.

12. Zou S, Shen Q, Hua Y, Jiang W, Zhang W, Zhu X. Proteomic identification of neoadjuvant chemotherapy-related proteins in bulky stage IB-IIA squamous cervical cancer. Reprod Sci. 2013;20(11):1356-1364.

13. Subramanian A, Tamayo P, Mootha VK, et al. Gene set enrichment analysis: a knowledge-based approach for interpreting genome-wide expression profiles. Proc Natl Acad Sci U S A. 2005;102(43):15545-15550.

14. Bader GD, Hogue CW. An automated method for finding molecular complexes in large protein interaction networks. BMC Bioinformatics. $2003 ; 4: 2$.

15. Hoskins ER, Hood BL, Sun M, Krivak TC, Edwards RP, Conrads TP. Proteomic analysis of ovarian cancer proximal fluids: validation of elevated peroxiredoxin 1 in patient peripheral circulation. PLoS One. 2011;6(9):e25056.

16. Duan J, Lang Y, Song C, Xiong J, Wang Y, Yan Y. siRNA targeting of PRDX3 enhances cisplatin-induced apoptosis in ovarian cancer cells through the suppression of the NF- $\kappa \mathrm{B}$ signaling pathway. Mol Med Rep. 2013;7(5):1688-1694.

17. Pak JH, Choi WH, Lee HM, et al. Peroxiredoxin 6 overexpression attenuates cisplatin-induced apoptosis in human ovarian cancer cells. Cancer Invest. 2011;29(1):21-28.

18. He T, Banach-Latapy A, Vernis L, Dardalhon M, Chanet R, Huang ME. Peroxiredoxin 1 knockdown potentiates $\beta$-lapachone cytotoxicity through modulation of reactive oxygen species and mitogen-activated protein kinase signals. Carcinogenesis. 2013;34(4):760-769.

19. Wend P, Holland JD, Ziebold U, Birchmeier W. Wnt signaling in stem and cancer stem cells. Semin Cell Dev Biol. 2010;21(8):855-863.

20. Talbot LJ, Bhattacharya SD, Kuo PC. Epithelial-mesenchymal transition, the tumor microenvironment, and metastatic behavior of epithelial malignancies. Int J Biochem Mol Biol. 2012;3(2):117-136.

21. Ha B, Kim EK, Kim JH, et al. Human peroxiredoxin 1 modulates TGF$\beta 1$-induced epithelial-mesenchymal transition through its peroxidase activity. Biochem Biophys Res Commun. 2012;421(1):33-37.

22. Yerlikaya A, Yontem M. Significance of Ubiquitin Proteasome Pathway in Cancer. Recent Pat Anticancer Drug Discov. 2013;8(3):298-309.

23. Guo QJ, Mills JN, Bandurraga SG, et al. MicroRNA-510 promotes cell and tumor growth by targeting peroxiredoxin 1 in breast cancer. Breast Cancer Res. 2013;15(4):R70.

24. Lee W, Choi KS, Riddell J, et al. Human peroxiredoxin 1 and 2 are not duplicate proteins: the unique presence of CYS83 in Prx1 underscores the structural and functional differences between Prx1 and Prx2. J Biol Chem. 2007;282(30):22011-22022.

25. Turner-Ivey B, Manevich Y, Schulte J, et al. Role for Prdx 1 as a specific sensor in redox-regulated senescence in breast cancer. Oncogene. 2013;32(45):5302-5314.

26. Taniuchi K, Furihata M, Hanazaki K, et al. Peroxiredoxin 1 promotes pancreatic cancer cell invasion by modulating p38 MAPK activity. Pancreas. 2015;44(2):331-340.
OncoTargets and Therapy

\section{Publish your work in this journal}

OncoTargets and Therapy is an international, peer-reviewed, open access journal focusing on the pathological basis of all cancers, potential targets for therapy and treatment protocols employed to improve the management of cancer patients. The journal also focuses on the impact of management programs and new therapeutic agents and protocols on

\section{Dovepress}

patient perspectives such as quality of life, adherence and satisfaction. The manuscript management system is completely online and includes a very quick and fair peer-review system, which is all easy to use. Visit http://www.dovepress.com/testimonials.php to read real quotes from published authors. 